\title{
The last of the seven-parton tree amplitudes
}

\author{
Daniel de Florian* and José Zurita ${ }^{\dagger}$ \\ Departamento de Física, FCEYN, Universidad de Buenos Aires, \\ (1428) Pabellón 1 Ciudad Universitaria, Capital Federal, Argentina
}

\begin{abstract}
We compute the four-quark plus three-gluon and six-quark plus one-gluon tree level amplitudes using on-shell recursion relations. They are needed for the calculation of the 5-jet cross-section at the Born level, and constitute an essential ingredient for next-to-leading order 4-jet and next-to-next-to-leading order 3-jet production at hadronic colliders. Very compact expressions for all possible helicity configurations are provided, allowing direct implementation in computer codes. With the results presented in this paper, the full set of seven-parton tree amplitudes becomes available.
\end{abstract}

August 2006

*email address: deflo@df.uba.ar

†email address: jzurita@df.uba.ar 


\section{Introduction}

With the forthcoming of the Large Hadronic Collider (LHC), the possibility to explore a wide range of new phenomena, like Higgs production, dark matter candidates, SUSY and new quark flavors will open. In order to study new physics, accurate calculations of cross-sections involving many partons are needed, for both signal and background. Such computation involves typically of the order of a thousand Feynman diagrams, so many that traditional methods become obsolete. During the 80 's, some new ideas were introduced [1-7] to allow a great simplification of the calculus, making possible the implementation of automatic computer algorithms (like MadEvent [8]) to perform this task. However, that still implies a huge computational effort, and the calculation of cross-sections for processes involving many partons remains as an unsolved problem.

In the last few years, the situation has drastically changed. Witten first noted the duality between tree level amplitudes and twistor string theory [9]. This lead to the introduction of the off-shell recursion relations by Cachazo, Svrček and Witten [10] (CSW), allowing to compute amplitudes for several partons by iterating the simplest MHV (Maximally Helicity Violating) ones [11]. Britto, Cachazo and Feng presented, as an ansatz, the onshell recursion relations [12], which were later proved to be correct (BCFW) [13]. The method was found very useful to provide compact expressions for gluon amplitudes. Soon it was extended to include massless fermions [14,15], Higgs boson [16], massive gauge bosons [17], photons [18] and massive fermions [19].

At tree level, six-parton processes were studied long time ago [20,21], and many of the amplitudes have been recomputed using the recursion relations recently [22,23]. Seven-gluon amplitudes were first calculated in [24], while certain eight-gluon results can be found in $[12,25]$. The two-fermion plus five-gluon amplitudes were presented in our previous paper [26]. At the loop level, the recursion relations become more complicated. However, calculations have reached the level of one-loop six-gluon amplitudes, and also some results for certain helicity configurations with an arbitrary number of partons were presented [27-38].

Recent work [39,40] also focused on the numerical implementation of the on-shell recursion relations. The result turns out to be that for a large number of partons, the "old" Berends-Giele [6] recursion relation performs better than the twistor inspired methods. Nevertheless, counting with analytical results for non trivial amplitudes would certainly speed up numerical calculations, reducing considerably the CPU-time required by any automatic algorithm. Therefore, obtaining very simple and compact results for the amplitudes is strongly desirable.

In this paper we present all four-quark plus three-gluon and six-quark plus one-gluon tree level helicity amplitudes, searching always for their most compact expressions. Our results have been checked against the factorization properties of the amplitudes, which provides a very strong consistency test. Combined with previous calculations $[12,26]$, the full set of seven-parton tree level amplitudes is now available.

This article is organized as follows: in Section 2 we review the color decomposition of tree level processes, and the main ingredients of the BCFW formulation, recalling its limitations when dealing with fermions. In Sections 3 and 4 we introduce the main results for the complete set of seven-parton amplitudes with two and three quarkantiquark pairs, respectively, while in Section 5 we present our conclusions. The necessary six-parton formulae for our calculation are collected in the appendix.

\section{Color decomposition, helicity and BCFW}

As is well known in the QCD literature, the color factor for the $m q \bar{q}$ pair plus $n$ gluon amplitude is [1]

$$
\Lambda\left(\left\{n_{i}\right\},\{\alpha\}\right)=\frac{(-1)^{p}}{N^{p}}\left(T^{a_{1}} \ldots T^{a_{n_{1}}}\right)_{i_{1} \alpha_{1}}\left(T^{a_{n_{1}+1}} \ldots T^{a_{n_{2}}}\right)_{i_{2} \alpha_{2}} \ldots\left(T^{a_{n_{m-1}+1}} \ldots T^{a_{n}}\right)_{i_{m} \alpha_{m}}
$$

where $i_{1}, \ldots, i_{m}$ are the color indices of the quarks and $\alpha_{1}, \ldots, \alpha_{m}$ are the ones for the antiquarks. The indices $n_{1}, \ldots, n_{m-1}$ (with $1 \leq n_{i} \leq n$ ) correspond to an arbitrary partition of an arbitrary permutation of the $n$ gluon indices. Each external quark is connected by a fermionic line to an external antiquark. When we want to indicate that a quark with color index $i_{k}$ and an antiquark with color index $\alpha_{k}$ are in fact connected by a fermionic line, we identify the index $\alpha_{k}$ with the index $\bar{i}_{k}$. Then the string $\{\alpha\}=\left(\alpha_{1}, \ldots, \alpha_{m}\right)$ is a generic permutation of the string $\bar{i}=\left(\bar{i}_{1}, \ldots, \bar{i}_{m}\right)$. The power $p$ is determined by the number of times $\alpha_{k}=\bar{i}_{k}$. If $\alpha \equiv \bar{i}$, then $p=m-1$. As 
usual, a product of zero $T$ matrices has to be understood as a Kronecker delta.

The full amplitude typically reads

$$
M_{n+m}^{(0)}=\sum_{\left\{n_{k}\right\},\left\{\alpha_{k}\right\}} \Lambda(\{n\},\{\alpha\}) A_{n+m}^{(0)}(\{i\},\{n\},\{\alpha\}),
$$

where the $A_{n+m}^{(0)}$ are known as the partial amplitudes. They depend only on the kinematic of the process, namely, the momenta and helicities of the particles.

In the framework of the helicity formalism [3-5], with the spinors denoted as

$$
\left|i^{ \pm}\right\rangle=\left|k_{i}^{ \pm}\right\rangle=\psi_{ \pm}\left(k_{i}\right) \quad\left\langle i^{ \pm}\right|=\left\langle k_{i}^{ \pm}\right|=\overline{\psi_{ \pm}\left(k_{i}\right)},
$$

the partial amplitudes can be written in terms of the spinors inner-products

$$
\begin{aligned}
& \langle i j\rangle=\left\langle i^{-} \mid j^{+}\right\rangle=\overline{\psi_{-}}\left(k_{i}\right) \psi_{+}\left(k_{j}\right) \\
& {[i j]=\left\langle i^{+} \mid j^{-}\right\rangle=\overline{\psi_{+}}\left(k_{i}\right) \psi_{-}\left(k_{j}\right),}
\end{aligned}
$$

and a few simple combinations of them, like

$$
\begin{array}{r}
\left\langle i\left|p_{a}\right| j\right] \equiv\langle i a\rangle[a j] \\
\left\langle i\left|p_{a} p_{b}\right| j\right\rangle \equiv\langle i a\rangle[a b]\langle b j\rangle .
\end{array}
$$

In our convention all particles are considered to be outgoing and, following the QCD literature [1,2], we fix the sign of the inner products such that $\langle i j\rangle[j i]=s_{i j}{ }^{\dagger}$.

The BCFW recurrence relation is based on the analytical properties of the amplitude when the spinors of two external legs (denoted by $j$ and $l$ ) are shifted as

$$
\begin{aligned}
& |\hat{j}\rangle=|j\rangle \\
& \mid \hat{j}]=\mid j]-z \mid l] \\
& |\hat{l}\rangle=|l\rangle+z|j\rangle \\
& \mid \hat{l}]=\mid l] .
\end{aligned}
$$

After this shift, the BCFW formula simply reads

$$
\begin{gathered}
A_{n}^{(0)}\left(1^{\lambda_{1}}, \ldots, n^{\lambda_{n}}\right)=\sum_{r, s} \sum_{\lambda= \pm} A_{s-r+2}^{(0)}\left(r^{\lambda_{r}}, \ldots, \hat{j}^{\lambda_{j}}, \ldots, s,-\hat{K}_{r s}^{\lambda}\right) \\
\quad \times \frac{1}{K_{r s}^{2}} A_{n-s+r}^{(0)}\left(\hat{K}_{r s}^{-\lambda},(s+1)^{\lambda_{(s+1)}}, \ldots, \hat{l}^{\lambda_{l}}, \ldots,(r-1)^{\lambda_{(r-1)}}\right),
\end{gathered}
$$

where $K_{r s}=k_{r}+\ldots+k_{j} \ldots+k_{s}$ and the (complex) shift variable $z$ takes the value

$$
z_{r s}=-\frac{\left(K_{r s}\right)^{2}}{\left\langle j\left|K_{r s}\right| l\right]}
$$

The sum on Eq.(7) is over all the possible configurations where the $j$ particle belongs to one of the amplitudes and the $l$ particle to the other one. It includes also the 3-particle sub-amplitudes, which are usually vanishing for on-shell particles but become non zero because of the BCFW shift in Eq. [6].

When tagging the particles, one has to fulfill certain restrictions, i.e.: some configurations of $j$ and $l$ are forbidden. The helicities of the reference particles can not be chosen as $\left(\lambda_{j}, \lambda_{l}\right)=(+,-)$.

Quarks and antiquarks of the same flavor can not be selected if they are adjacent. Furthermore, one can not chose two adjacent fermions of different flavor and the same helicity. For adjacent quarks and gluons the helicities should better be opposite. [22].

\footnotetext{
${ }^{\dagger}$ When comparing with results obtained using the string-like conventions just notice that $[i j]$ carries the opposite sign
} 
Under a general tag, some sub-amplitudes may vanish. A clever choice of $j$ and $l$ leads to most compact expressions for the same amplitudes. In this paper, we have chosen the allowed tag that gives the minor number of terms. Nevertheless, in some calculations we have tried some forbidden tags, in the search for results with fewer terms and simpler structure. In that case we explicitly checked that the final expressions agreed with the longer forms obtained using allowed legs in the BCFW recursion relation.

The main ingredient of the recursion relations, that permits to construct the tree level amplitude for $n$-partons just by iterations, are the $g g g$ and $q \bar{q} g$ MHV amplitudes [11], which with our phase conventions read

$$
\begin{gathered}
A_{3}^{(0)}\left(1_{g}^{+}, 2_{g}^{-}, 3_{g}^{-}\right)=\frac{\langle 23\rangle^{3}}{\langle 12\rangle\langle 31\rangle}, \\
A_{3}^{(0)}\left(1_{q}^{+}, 2_{\bar{q}}^{-}, 3_{g}^{-}\right)=\frac{\langle 23\rangle^{2}}{\langle 21\rangle} .
\end{gathered}
$$

The corresponding $\overline{\mathrm{MHV}}$ amplitudes can be obtained from those above by using some simple properties. The "parity" operation inverts the helicities of all the particles, and at the level of the amplitudes implies to perform the exchange $\langle i j\rangle \leftrightarrow[j i]$ and add an extra factor of $(-1)$ for each fermionic pair involved. Charge conjugation turns quarks into antiquarks and vice-versa. The partial amplitudes are C-invariant: they do not change under the action of this operator. Also one can profit from the reflection and cyclic symmetries of the amplitudes. The reflection property states that the following equality holds

$$
A(1,2, \ldots, n-1, n)=(-1)^{n} A(n, n-1, \ldots, 2,1),
$$

while cyclic symmetry implies that the amplitude is invariant under cyclic permutations of $1,2, \ldots, n$. The factorization properties of the amplitudes in the collinear and soft channels are an useful tool to check for the consistency of the results. Further details on the method of calculation can be found in [26].

\section{Four-quark plus three-gluon amplitudes}

Seeking for the most general presentation, we concentrate on the case of different quark flavors, that we denote as $q$ and $p$. To obtain the equal flavor two-quark pair amplitudes, it is enough to substract from a given partial amplitude the same term with the antiquarks exchanged (and not the quarks) [20,41].

From Eq.(1), we can read off the two different color structures contributing to the two-quark pair plus gluon amplitudes. For $p=0$, it follows $\alpha_{1}=\bar{i}_{2}, \alpha_{2}=\bar{i}_{1}$ and one obtains the leading contribution

$$
\left(T^{a\left(\sigma_{1}\right)} \ldots T^{a\left(\sigma_{j}\right)}\right)_{i_{1} \bar{i}_{2}}\left(T^{a\left(\sigma_{j+1}\right)} \ldots T^{a\left(\sigma_{l}\right)}\right)_{i_{2} \bar{i}_{1}},
$$

while if $p=1$ (in this case $\alpha \equiv \bar{i}$ ) there is a sub-leading term

$$
\frac{-1}{N}\left(T^{a\left(\sigma_{1}\right)} \ldots T^{a\left(\sigma_{j}\right)}\right)_{i_{1} \bar{i}_{1}}\left(T^{a\left(\sigma_{j+1}\right)} \ldots T^{a\left(\sigma_{l}\right)}\right)_{i_{2} \bar{i}_{2}} .
$$

For leading color, the partial amplitude corresponds to the following string of quarks and gluons

$$
\left[q\left(g_{\sigma_{1}} \ldots g_{\sigma_{j}}\right) \bar{p}\right]\left[p\left(g_{\sigma_{j+1}} \ldots g_{\sigma_{l}}\right) \bar{q}\right]
$$

while for the sub-leading color the ordering is

$$
\left[q\left(g_{\sigma_{1}} \ldots g_{\sigma_{j}}\right) \bar{q}\right]\left[p\left(g_{\sigma_{j+1}} \ldots g_{\sigma_{l}}\right) \bar{p}\right] .
$$

In order to maintain a similar notation (the first particle being always $q$ ) to the one used in [26] for one-quark pair amplitudes, we will present the results with the following format

$$
A\left(q, \bar{q}, g_{\sigma_{1}}, \ldots, g_{\sigma_{j}}, p, \bar{p}, g_{\sigma_{j+1}}, \ldots, g_{\sigma_{l}}\right)
$$

and

$$
A\left(q, \bar{p}, g_{\sigma_{1}}, \ldots, g_{\sigma_{j}}, p, \bar{q}, g_{\sigma_{j+1}}, \ldots, g_{\sigma_{l}}\right)
$$


for the leading and sub-leading color structures, respectively. Those corresponding exactly to the ordering in Eq.(13) and Eq.(14) can be easily obtained from the ones above by $C$ operation and relabelling of the partons under cyclic symmetry.

Several amplitudes are accounted for by the MHV formula for two-quark pairs and $n-4$ gluons, which is given by

$$
A_{n}^{(0)}\left(1_{q}, 2_{\bar{q}}, 3_{g}, \ldots, k_{g},(k+1)_{p},(k+2)_{\bar{p}},(k+3)_{g}, \ldots, n_{g}\right)=\frac{(-1)\langle i j\rangle^{2}\langle p \bar{q}\rangle\langle q \bar{p}\rangle}{\prod_{l=1}^{n}\langle l l+1\rangle},
$$

where $i$ and $j$ are the two fermions with negative helicity while all the other particles have positive helicity [1]. We have reobtained Eq.(17) using the BCFW recursion relation and Eq. (9). For the sub-leading case exactly the same formula holds.

When facing the NHMV case, we denote the leading and sub-leading color contributions as "A" and "B", respectively. There are fourteen independent "A" amplitudes and twelve independent "B" amplitudes. For simplicity, we will refer to them as $A_{1}, A_{2}, \ldots, A_{14}$ and $B_{1}, B_{2}, \ldots, B_{12}$. The full list is

$$
\begin{array}{ll}
A_{1}=A_{7}^{(0)}\left(1_{q}^{+}, 2_{\bar{q}}^{-}, 3^{+}, 4^{+}, 5^{-}, 6_{p}^{+}, 7_{\bar{p}}^{-}\right) & A_{8}=A_{7}^{(0)}\left(1_{q}^{+}, 2_{\bar{q}}^{-}, 3^{-}, 4^{+}, 5_{p}^{+}, 6_{\bar{p}}^{-}, 7^{+}\right) \\
A_{2}=A_{7}^{(0)}\left(1_{q}^{+}, 2_{\bar{q}}^{-}, 3^{+}, 4^{-}, 5^{+}, 6_{p}^{+}, 7_{\bar{p}}^{-}\right) & A_{9}=A_{7}^{(0)}\left(1_{q}^{+}, 2_{\bar{q}}^{-}, 3^{+}, 4^{+}, 5_{p}^{-}, 6_{\bar{p}}^{+}, 7^{-}\right) \\
A_{3}=A_{7}^{(0)}\left(1_{q}^{+}, 2_{\bar{q}}^{-}, 3^{-}, 4^{+}, 5^{+}, 6_{p}^{+}, 7_{\bar{p}}^{-}\right) & A_{10}=A_{7}^{(0)}\left(1_{q}^{+}, 2_{\bar{q}}^{-}, 3^{+}, 4^{-}, 5_{p}^{-}, 6_{\bar{p}}^{+}, 7^{+}\right) \\
A_{4}=A_{7}^{(0)}\left(1_{q}^{+}, 2_{\bar{q}}^{-}, 3^{+}, 4^{+}, 5^{-}, 6_{p}^{-}, 7_{\bar{p}}^{+}\right) & A_{11}=A_{7}^{(0)}\left(1_{q}^{-}, 2_{\bar{q}}^{+}, 3^{+}, 4^{+}, 5^{-}, 6_{p}^{+}, 7_{\bar{p}}^{-}\right) \\
A_{5}=A_{7}^{(0)}\left(1_{q}^{+}, 2_{\bar{q}}^{-}, 3^{+}, 4^{-}, 5^{+}, 6_{p}^{-}, 7_{\bar{p}}^{+}\right) & A_{12}=A_{7}^{(0)}\left(1_{q}^{-}, 2_{\bar{q}}^{+}, 3^{+}, 4^{-}, 5^{+}, 6_{p}^{+}, 7_{\bar{p}}^{-}\right) \\
A_{6}=A_{7}^{(0)}\left(1_{q}^{+}, 2_{\bar{q}}^{-}, 3^{+}, 4^{+}, 5_{p}^{+}, 6_{\bar{p}}^{-}, 7^{-}\right) & A_{13}=A_{7}^{(0)}\left(1_{q}^{-}, 2_{\bar{q}}^{+}, 3^{+}, 4^{+}, 5_{p}^{+}, 6_{\bar{p}}^{-}, 7^{-}\right) \\
A_{7}=A_{7}^{(0)}\left(1_{q}^{+}, 2_{\bar{q}}^{-}, 3^{+}, 4^{-}, 5_{p}^{+}, 6_{\bar{p}}^{-}, 7^{+}\right) & A_{14}=A_{7}^{(0)}\left(1_{\bar{q}}^{-}, 2_{\bar{q}}^{+}, 3^{+}, 4^{-}, 5_{p}^{+}, 6_{\bar{p}}^{-}, 7^{+}\right)
\end{array}
$$

for type A processes and

$$
\begin{array}{ll}
B_{1}=A_{7}^{(0)}\left(1_{q}^{+}, 2_{\bar{p}}^{-}, 3^{+}, 4^{+}, 5^{-}, 6_{p}^{+}, 7_{\bar{q}}^{-}\right) & B_{7}=A_{7}^{(0)}\left(1_{q}^{+}, 2_{\bar{p}}^{-}, 3^{+}, 4^{+}, 5_{p}^{+}, 6_{\bar{q}}^{-}, 7^{-}\right) \\
B_{2}=A_{7}^{(0)}\left(1_{q}^{+}, 2_{\bar{p}}^{-}, 3^{+}, 4^{-}, 5^{+}, 6_{p}^{+}, 7_{\bar{q}}^{-}\right) & B_{8}=A_{7}^{(0)}\left(1_{q}^{+}, 2_{\bar{p}}^{-}, 3^{+}, 4^{-}, 5_{p}^{+}, 6_{\bar{q}}^{-}, 7^{+}\right) \\
B_{3}=A_{7}^{(0)}\left(1_{q}^{+}, 2_{\bar{p}}^{-}, 3^{-}, 4^{+}, 5^{+}, 6_{p}^{+}, 7_{\bar{q}}^{-}\right) & B_{9}=A_{7}^{(0)}\left(1_{q}^{+}, 2_{\bar{p}}^{-}, 3^{-}, 4^{+}, 5_{p}^{+}, 6_{\bar{q}}^{-}, 7^{+}\right) \\
B_{4}=A_{7}^{(0)}\left(1_{q}^{+}, 2_{\bar{p}}^{+}, 3^{+}, 4^{+}, 5^{-}, 6_{p}^{-}, 7_{\bar{q}}^{-}\right) & B_{10}=A_{7}^{(0)}\left(1_{q}^{+}, 2_{\bar{p}}^{+}, 3^{+}, 4^{+}, 5_{p}^{-}, 6_{\bar{q}}^{-}, 7^{-}\right) \\
B_{5}=A_{7}^{(0)}\left(1_{q}^{+}, 2_{\bar{p}}^{+}, 3^{+}, 4^{-}, 5^{+}, 6_{p}^{-}, 7_{\bar{q}}^{-}\right) & B_{11}=A_{7}^{(0)}\left(1_{q}^{+}, 2_{\bar{p}}^{+}, 3^{+}, 4^{-}, 5_{p}^{-}, 6_{\bar{q}}^{-}, 7^{+}\right) \\
B_{6}=A_{7}^{(0)}\left(1_{q}^{+}, 2_{\bar{p}}^{+}, 3^{-}, 4^{+}, 5^{+}, 6_{p}^{-}, 7_{\bar{q}}^{-}\right) & B_{12}=A_{7}^{(0)}\left(1_{q}^{+}, 2_{\bar{p}}^{+}, 3^{-}, 4^{+}, 5_{p}^{-}, 6_{\bar{q}}^{-}, 7^{+}\right)
\end{array}
$$

for type B processes.

The explicits results for those amplitudes are presented in the next sub-sections.

\section{$3.14 q+3 g$ leading amplitudes}

We present now the most compact expressions for the 14 independent leading amplitudes. 
We have taken $j=5, l=6$ for the calculation of $A_{1}$, leading to

$$
\begin{aligned}
A_{1} & =A_{7}^{(0)}\left(1_{q}^{+}, 2_{\bar{q}}^{-}, 3^{+}, 4^{+}, 5^{-}, 6_{p}^{+}, 7_{\bar{p}}^{-}\right)= \\
& +\frac{\langle 57\rangle^{3}\langle 2|3+1| 4]^{3}}{s_{567} s_{123}\langle 12\rangle\langle 23\rangle\langle 67\rangle\langle 7|6+5| 4]\langle 3|(2+1)(7+6)| 5\rangle} \\
& +\frac{\langle 57\rangle^{3}\langle 5|4+3| 1]^{3}}{[12]\langle 34\rangle\langle 45\rangle\langle 67\rangle\langle 5|6+7| 1]\langle 3|(2+1)(7+6)| 5\rangle\langle 5|(4+3)(2+1)| 7\rangle} \\
& -\frac{[61]^{2}\langle 25\rangle^{3}\langle 5|1+7| 6]}{s_{671}\langle 23\rangle\langle 34\rangle\langle 45\rangle[67]\langle 2|1+7| 6]\langle 5|6+7| 1]} \\
& -\frac{\langle 27\rangle^{2}\langle 5|4+3| 6]^{3}\langle 5|(4+3)(1+7)| 2\rangle}{s_{712} s_{345}\langle 12\rangle\langle 34\rangle\langle 45\rangle\langle 3|4+5| 6]\langle 2|1+7| 6]\langle 5|(4+3)(2+1)| 7\rangle} \\
& +\frac{\langle 27\rangle^{2}[46]^{3}\langle 2|6+5| 4]}{s_{456}\langle 12\rangle\langle 23\rangle[45][56]\langle 3|4+5| 6]\langle 7|6+5| 4]} .
\end{aligned}
$$

$A_{2}$ has been calculated by selecting $j=7, l=1$, obtaining

$$
\begin{aligned}
A_{2} & =A_{7}^{(0)}\left(1_{q}^{+}, 2_{\bar{q}}^{-}, 3^{+}, 4^{-}, 5^{+}, 6_{p}^{+}, 7_{\bar{p}}^{-}\right)= \\
& +\frac{\langle 46\rangle\langle 27\rangle^{2}\langle 2|1+7| 3]\langle 4|5+6| 3]^{3}}{s_{456} s_{712}\langle 12\rangle\langle 45\rangle\langle 56\rangle\langle 6|5+4| 3]\langle 7|1+2| 3]\langle 2|(1+7)(6+5)| 4\rangle} \\
& +\frac{\langle 27\rangle^{2}\langle 24\rangle^{4}[56]^{2}\langle 2|1+7| 5]}{\langle 12\rangle\langle 23\rangle\langle 34\rangle\langle 2|3+4| 5]\langle 2|1+7| 6]\langle 7|(6+5)(4+3)| 2\rangle\langle 2|(1+7)(6+5)| 4\rangle} \\
& -\frac{\langle 26\rangle\langle 27\rangle^{2}[35]^{4}}{s_{345}\langle 12\rangle[34][45]\langle 67\rangle\langle 2|3+4| 5]\langle 6|5+4| 3]} \\
& -\frac{\langle 46\rangle\langle 47\rangle^{3}[13]^{3}}{s_{123}[12]\langle 45\rangle\langle 56\rangle\langle 67\rangle\langle 4|3+2| 1]\langle 7|1+2| 3]} \\
& -\frac{\langle 24\rangle^{3}\langle 6|5+7| 1]\langle 7|6+5| 1]^{2}\langle 7|(6+5)(2+3)| 4\rangle}{s_{234} s_{567}\langle 23\rangle\langle 34\rangle\langle 56\rangle\langle 67\rangle\langle 5|6+7| 1]\langle 4|3+2| 1]\langle 7|(6+5)(4+3)| 2\rangle} \\
& -\frac{[16]^{2}\langle 24\rangle^{3}\langle 4|1+7| 6]}{s_{671}\langle 23\rangle\langle 34\rangle\langle 45\rangle[67]\langle 2|1+7| 6]\langle 5|6+7| 1]} .
\end{aligned}
$$

Choosing $j=4, l=5, A_{3}$ is given by

$$
\begin{aligned}
A_{3} & =A_{7}^{(0)}\left(1_{q}^{+}, 2_{\bar{q}}^{-}, 3^{-}, 4^{+}, 5^{+}, 6_{p}^{+}, 7_{\bar{p}}^{-}\right)= \\
& -\frac{\langle 27\rangle^{2} s_{456}^{2}\langle 6|(5+4)(7+1)| 2\rangle}{s_{712}\langle 12\rangle\langle 45\rangle\langle 56\rangle\langle 6|5+4| 3]\langle 7|1+2| 3]\langle 2|(1+7)(6+5)| 4\rangle} \\
& -\frac{\langle 6|3+2| 1]\langle 7|1+3| 2]\langle 7|3+2| 1]^{2}}{s_{123}[12][23]\langle 45\rangle\langle 56\rangle\langle 67\rangle\langle 4|3+2| 1]\langle 7|1+2| 3]} \\
& +\frac{\langle 46\rangle\langle 23\rangle^{2}\langle 4|5+6| 1]^{2}\langle 3|(7+1)(6+5)| 4\rangle}{\langle 34\rangle\langle 45\rangle\langle 56\rangle\langle 4|5+6| 7]\langle 4|3+2| 1]\langle 6|(7+1)(2+3)| 4\rangle\langle 2|(1+7)(6+5)| 4\rangle} \\
& -\frac{\langle 13\rangle\langle 23\rangle^{2}[57][56]^{2}}{s_{567}\langle 12\rangle\langle 34\rangle[67]\langle 4|5+6| 7]\langle 1|7+6| 5]} \\
& -\frac{\langle 23\rangle^{2}\langle 3|2+4| 5]\langle 4|3+2| 5]\langle 6|7+1| 5]\langle 7|1+6| 5]^{2}}{s_{671} s_{234}\langle 34\rangle\langle 67\rangle\langle 2|3+4| 5]\langle 4|3+2| 5]\langle 1|7+6| 5]\langle 6|(7+1)(2+3)| 4\rangle} \\
& -\frac{\langle 26\rangle\langle 27\rangle^{2}[45]^{3}}{s_{345}\langle 12\rangle[34]\langle 67\rangle\langle 2|3+4| 5]\langle 6|5+4| 3]} .
\end{aligned}
$$


Picking $j=3, l=4$ we have obtained for $A_{4}$ the following expression

$$
\begin{aligned}
A_{4} & =A_{7}^{(0)}\left(1_{q}^{+}, 2_{\bar{q}}^{-}, 3^{+}, 4^{+}, 5^{-}, 6_{p}^{-}, 7_{\bar{p}}^{+}\right)= \\
& -\frac{[17]^{2}\langle 25\rangle^{3}\langle 5|1+7| 6]}{s_{671}\langle 23\rangle\langle 34\rangle\langle 45\rangle[67]\langle 5|6+7| 1]\langle 2|1+7| 6]} \\
& -\frac{\langle 5|4+3| 6]\langle 2|(1+7)(3+4)| 5\rangle^{3}}{s_{712} s_{345}\langle 12\rangle\langle 34\rangle\langle 45\rangle\langle 3|4+5| 6]\langle 2|1+7| 6]\langle 7|(1+2)(3+4)| 5\rangle} \\
& +\frac{\langle 57\rangle\langle 56\rangle^{2}\langle 5|4+3| 1]^{3}}{[12]\langle 34\rangle\langle 45\rangle\langle 67\rangle\langle 5|6+7| 1]\langle 5|(6+7)(1+2)| 3\rangle\langle 7|(1+2)(3+4)| 5\rangle} \\
& +\frac{[46]\langle 2|6+5| 4]^{3}}{s_{456}\langle 12\rangle\langle 23\rangle[45][56]\langle 7|6+5| 4]\langle 3|4+5| 6]} \\
& -\frac{\langle 57\rangle\langle 56\rangle^{2}\langle 2|1+3| 4]^{3}}{s_{567} s_{123}\langle 12\rangle\langle 23\rangle\langle 67\rangle\langle 7|6+5| 4]\langle 5|(6+7)(1+2)| 3\rangle} .
\end{aligned}
$$

$A_{5}$ has been calculated by taking $j=4, l=5$. This gives

$$
\begin{aligned}
A_{5} & =A_{7}^{(0)}\left(1_{q}^{+}, 2_{\bar{q}}^{-}, 3^{+}, 4^{-}, 5^{+}, 6_{p}^{-}, 7_{\bar{p}}^{+}\right)= \\
& -\frac{[17]^{2}\langle 24\rangle^{3}\langle 46\rangle^{3}\langle 4|(5+6)(3+2)| 4\rangle}{\langle 23\rangle\langle 34\rangle\langle 45\rangle\langle 56\rangle\langle 4|5+6| 7]\langle 4|3+2| 1]\langle 4|(5+6)(7+1)| 2\rangle\langle 6|(7+1)(2+3)| 4\rangle} \\
& -\frac{\langle 46\rangle^{3}\langle 4|5+6| 3]\langle 2|1+7| 3]^{3}}{s_{456} s_{712}\langle 12\rangle\langle 45\rangle\langle 56\rangle\langle 6|5+4| 3]\langle 7|1+2| 3]\langle 4|(5+6)(7+1)| 2\rangle} \\
& -\frac{\langle 47\rangle\langle 46\rangle^{3}[13]^{3}}{s_{123}[12]\langle 45\rangle\langle 56\rangle\langle 67\rangle\langle 7|1+2| 3]\langle 4|3+2| 1]} \\
& -\frac{\langle 14\rangle\langle 24\rangle^{3}[57]^{3}}{s_{567}\langle 12\rangle\langle 23\rangle\langle 34\rangle[67]\langle 4|5+6| 7]\langle 1|7+6| 5]} \\
& -\frac{\langle 24\rangle^{3}\langle 4|3+2| 5]\langle 6|7+1| 5]^{3}}{s_{671} s_{234}\langle 23\rangle\langle 34\rangle\langle 67\rangle\langle 2|3+4| 5]\langle 1|7+6| 5]\langle 6|(7+1)(2+3)| 4\rangle} \\
& -\frac{\langle 26\rangle^{3}[35]^{4}}{s_{345}\langle 12\rangle[34][45]\langle 67\rangle\langle 2|3+4| 5]\langle 6|5+4| 3]} .
\end{aligned}
$$

$A_{6}$ was computed by choosing $j=7, l=1$, arriving at

$$
\begin{aligned}
A_{6} & =A_{7}^{(0)}\left(1_{q}^{+}, 2_{\bar{q}}^{-}, 3^{+}, 4^{+}, 5_{p}^{+}, 6_{\bar{p}}^{-}, 7^{-}\right)= \\
& -\frac{\langle 27\rangle^{3}\langle 5|6+4| 3]\langle 6|5+4| 3]^{2}}{s_{456} s_{712}\langle 12\rangle\langle 45\rangle\langle 56\rangle\langle 7|1+2| 3]\langle 2|(1+7)(6+5)| 4\rangle} \\
& +\frac{\langle 27\rangle^{3}\langle 2|3+4| 6]\langle 2|3+4| 5]^{2}}{\langle 12\rangle\langle 23\rangle\langle 34\rangle[56]\langle 2|1+7| 6]\langle 2|(3+4)(5+6)| 7\rangle\langle 2|(1+7)(6+5)| 4\rangle} \\
& -\frac{\langle 57\rangle\langle 67\rangle^{2}[13]^{3}}{s_{123}[12]\langle 45\rangle\langle 56\rangle\langle 4|3+2| 1]\langle 7|1+2| 3]} \\
& +\frac{\langle 57\rangle\langle 67\rangle^{2}\langle 2|3+4| 1]^{3}}{s_{567}\langle 23\rangle\langle 34\rangle\langle 56\rangle\langle 5|6+7| 1]\langle 4|3+2| 1]\langle 2|(3+4)(5+6)| 7\rangle} \\
& -\frac{\langle 25\rangle[16]\langle 2|6+7| 1]^{2}}{\langle 23\rangle\langle 34\rangle\langle 45\rangle[67][71]\langle 2|1+7| 6]\langle 5|6+7| 1]} .
\end{aligned}
$$


$A_{7}$ was obtained by picking $j=4, l=5$. The final result gives

$$
\begin{aligned}
A_{7} & =A_{7}^{(0)}\left(1_{q}^{+}, 2_{\bar{q}}^{-}, 3^{+}, 4^{-}, 5_{p}^{+}, 6_{\bar{p}}^{-}, 7^{+}\right)= \\
& +\frac{[17]^{2}\langle 24\rangle^{3}\langle 46\rangle^{3}\langle 4|3+2| 7]}{\langle 23\rangle\langle 34\rangle\langle 56\rangle\langle 4|5+6| 7]\langle 4|3+2| 1]\langle 6|(7+1)(2+3)| 4\rangle\langle 4|(5+6)(7+1)| 2\rangle} \\
& -\frac{\langle 46\rangle^{3}\langle 1|7+2| 3]\langle 2|1+7| 3]^{3}}{s_{456} s_{712}\langle 56\rangle\langle 71\rangle\langle 12\rangle\langle 6|5+4| 3]\langle 7|1+2| 3]\langle 4|(5+6)(7+1)| 2\rangle} \\
& -\frac{[13]^{3}\langle 46\rangle^{3}}{s_{123}[12]\langle 56\rangle\langle 67\rangle\langle 7|1+2| 3]\langle 4|3+2| 1]} \\
& -\frac{\langle 14\rangle\langle 24\rangle^{3}[57]^{3}}{s_{567}\langle 12\rangle\langle 23\rangle\langle 34\rangle[56]\langle 1|7+6| 5]\langle 4|5+6| 7]} \\
& -\frac{\langle 16\rangle\langle 24\rangle^{3}\langle 4|3+2| 5]\langle 6|7+1| 5]^{2}}{s_{234}\langle 23\rangle\langle 34\rangle\langle 67\rangle\langle 71\rangle\langle 2|3+4| 5]\langle 1|7+6| 5]\langle 6|(7+1)(2+3)| 4\rangle} \\
& -\frac{\langle 16\rangle\langle 26\rangle^{2}[35]^{3}\langle 2|5+4| 3]}{s_{345}\langle 67\rangle\langle 71\rangle\langle 12\rangle[34][45]\langle 2|3+4| 5]\langle 6|5+4| 3]} .
\end{aligned}
$$

We have computed $A_{8}$ by selecting $j=6, l=7$

$$
\begin{aligned}
A_{8} & =A_{7}^{(0)}\left(1_{q}^{+}, 2_{\bar{q}}^{-}, 3^{-}, 4^{+}, 5_{p}^{+}, 6_{\bar{p}}^{-}, 7^{+}\right)= \\
& -\frac{\langle 16\rangle\langle 26\rangle^{2}[45]^{2}\langle 2|3+5| 4]}{s_{345}\langle 12\rangle[34]\langle 67\rangle\langle 71\rangle\langle 2|3+4| 5]\langle 6|5+4| 3]} \\
& -\frac{\langle 16\rangle\langle 6|5+4| 2]\langle 6|(7+1)(2+3)| 5\rangle\langle 6|(7+1)(2+3)| 6\rangle^{2}}{[23]\langle 45\rangle\langle 56\rangle\langle 67\rangle\langle 71\rangle\langle 6|7+1| 2]\langle 6|5+4| 3]\langle 6|(5+4)(3+2)| 1\rangle\langle 6|(7+1)(2+3)| 4\rangle} \\
& -\frac{\langle 16\rangle\langle 23\rangle^{2}\langle 3|2+4| 5]\langle 6|7+1| 5]^{2}}{s_{234}\langle 34\rangle\langle 67\rangle\langle 71\rangle\langle 1|7+6| 5]\langle 2|3+4| 5]\langle 6|(7+1)(2+3)| 4\rangle} \\
& +\frac{\langle 35\rangle\langle 36\rangle^{3}[27][17]^{2}}{s_{712}[12]\langle 34\rangle\langle 45\rangle\langle 56\rangle\langle 6|7+1| 2]\langle 3|2+1| 7]} \\
& +\frac{\langle 13\rangle\langle 23\rangle^{2}\langle 5|4+6| 7]\langle 6|5+4| 7]^{3}}{s_{123} s_{456}\langle 12\rangle\langle 45\rangle\langle 56\rangle\langle 4|5+6| 7]\langle 3|2+1| 7]\langle 6|(5+4)(3+2)| 1\rangle} \\
& -\frac{\langle 13\rangle\langle 23\rangle^{2}[57]^{3}}{s_{567}\langle 12\rangle\langle 34\rangle[56]\langle 4|5+6| 7]\langle 1|7+6| 5]} .
\end{aligned}
$$

Choosing $j=2, l=3$, we arrive at the following expression for $A_{9}$

$$
\begin{aligned}
A_{9} & =A_{7}^{(0)}\left(1_{q}^{+}, 2_{\bar{q}}^{-}, 3^{+}, 4^{+}, 5_{p}^{-}, 6_{\bar{p}}^{+}, 7^{-}\right)= \\
& -\frac{\langle 25\rangle^{3}[16]^{3}}{\langle 23\rangle\langle 34\rangle\langle 45\rangle[67][71]\langle 5|6+7| 1]\langle 2|1+7| 6]} \\
& -\frac{\langle 27\rangle^{3}\langle 2|3+4| 6]^{3}}{\langle 12\rangle\langle 23\rangle\langle 34\rangle[56]\langle 2|1+7| 6]\langle 4|(5+6)(7+1)| 2\rangle\langle 2|(3+4)(5+6)| 7\rangle} \\
& +\frac{\langle 57\rangle^{3}\langle 2|3+4| 1]^{3}}{s_{567}\langle 23\rangle\langle 34\rangle\langle 56\rangle\langle 4|3+2| 1]\langle 5|6+7| 1]\langle 2|(3+4)(5+6)| 7\rangle} \\
& +\frac{\langle 27\rangle^{3}\langle 5|6+4| 3]^{3}}{s_{456} s_{712}\langle 12\rangle\langle 45\rangle\langle 56\rangle\langle 7|1+2| 3]\langle 4|(5+6)(7+1)| 2\rangle} \\
& -\frac{[13]^{3}\langle 57\rangle^{3}}{s_{123}[12]\langle 45\rangle\langle 56\rangle\langle 7|1+2| 3]\langle 4|3+2| 1]} .
\end{aligned}
$$


The computation of $A_{10}$ has been performed by taking $j=2, l=3$, leading to

$$
\begin{aligned}
A_{10} & =A_{7}^{(0)}\left(1_{q}^{+}, 2_{\bar{q}}^{-}, 3^{+}, 4^{-}, 5_{p}^{-}, 6_{\bar{p}}^{+}, 7^{+}\right)= \\
& +\frac{\langle 46\rangle\langle 45\rangle^{2}\langle 24\rangle^{3}[17]^{2}\langle 4|3+2| 7]}{\langle 23\rangle\langle 34\rangle\langle 56\rangle\langle 4|3+2| 1]\langle 4|5+6| 7]\langle 4|(5+6)(7+1)| 2\rangle\langle 6|(7+1)(2+3)| 4\rangle} \\
& -\frac{\langle 14\rangle\langle 24\rangle^{3}[57][67]^{2}}{s_{567}\langle 12\rangle\langle 23\rangle\langle 34\rangle[56]\langle 4|5+6| 7]\langle 1|7+6| 5]} \\
& -\frac{\left(s_{671}\right)^{2}\langle 16\rangle\langle 24\rangle^{3}\langle 4|3+2| 5]}{s_{234}\langle 23\rangle\langle 34\rangle\langle 67\rangle\langle 71\rangle\langle 2|3+4| 5]\langle 1|7+6| 5]\langle 6|(7+1)(2+3)| 4\rangle} \\
& -\frac{\langle 16\rangle[35]\langle 2|5+4| 3]^{3}}{s_{345}[34][45]\langle 67\rangle\langle 71\rangle\langle 12\rangle\langle 2|3+4| 5]\langle 6|5+4| 3]} \\
& -\frac{\langle 46\rangle\langle 45\rangle^{2}\langle 1|7+2| 3]\langle 2|1+7| 3]^{3}}{s_{456} s_{712}\langle 56\rangle\langle 71\rangle\langle 12\rangle\langle 7|1+2| 3]\langle 6|5+4| 3]\langle 4|(5+6)(7+1)| 2\rangle} \\
- & \frac{\langle 46\rangle\langle 45\rangle^{2}[13]^{3}}{s_{123}[12]\langle 56\rangle\langle 67\rangle\langle 4|3+2| 1]\langle 7|1+2| 3]} .
\end{aligned}
$$

We have selected $j=3, l=4$ for the calculation of $A_{11}$, arriving at

$$
\begin{aligned}
A_{11} & =A_{7}^{(0)}\left(1_{q}^{-}, 2_{\bar{q}}^{+}, 3^{+}, 4^{+}, 5^{-}, 6_{p}^{+}, 7_{\bar{p}}^{-}\right)= \\
& -\frac{\langle 71\rangle^{2}\langle 5|4+3| 6]^{3}\langle 2|(1+7)(3+4)| 5\rangle}{s_{712} s_{345}\langle 12\rangle\langle 34\rangle\langle 45\rangle\langle 2|1+7| 6]\langle 3|4+5| 6]\langle 7|(1+2)(3+4)| 5\rangle} \\
& -\frac{\langle 25\rangle\langle 5|1+7| 6]^{3}}{s_{671}\langle 23\rangle\langle 34\rangle\langle 45\rangle[67]\langle 2|1+7| 6]\langle 5|6+7| 1]} \\
& +\frac{\langle 57\rangle^{3}\langle 5|4+3| 1]\langle 5|4+3| 2]^{2}}{[12]\langle 34\rangle\langle 45\rangle\langle 67\rangle\langle 5|6+7| 1]\langle 5|(6+7)(1+2)| 3\rangle\langle 7|(1+2)(3+4)| 5\rangle} \\
& +\frac{\langle 71\rangle^{2}[46]^{3}\langle 2|6+5| 4]}{s_{456}\langle 12\rangle\langle 23\rangle[45][56]\langle 3|4+5| 6]\langle 7|6+5| 4]} \\
& -\frac{\langle 57\rangle^{3}\langle 2|1+3| 4]\langle 1|2+3| 4]^{2}}{s_{567} s_{123}\langle 12\rangle\langle 23\rangle\langle 67\rangle\langle 7|6+5| 4]\langle 5|(6+7)(1+2)| 3\rangle} .
\end{aligned}
$$

By choosing $j=4, l=5$, we have computed $A_{12}$

$$
\begin{aligned}
A_{12} & =A_{7}^{(0)}\left(1_{q}^{-}, 2_{\bar{q}}^{+}, 3^{+}, 4^{-}, 5^{+}, 6_{p}^{+}, 7_{\bar{p}}^{-}\right)= \\
& -\frac{\langle 46\rangle\langle 71\rangle^{2}\langle 2|1+7| 3]\langle 4|5+6| 3]^{3}}{s_{456} s_{712}\langle 12\rangle\langle 45\rangle\langle 56\rangle\langle 6|5+4| 3]\langle 7|1+2| 3]\langle 4|(5+6)(7+1)| 2\rangle} \\
& +\frac{\langle 24\rangle\langle 46\rangle\langle 4|(3+2)(6+5)| 4\rangle^{3}}{\langle 23\rangle\langle 34\rangle\langle 45\rangle\langle 56\rangle\langle 4|3+2| 1]\langle 4|5+6| 7]\langle 6|(7+1)(2+3)| 4\rangle\langle 4|(5+6)(7+1)| 2\rangle} \\
& -\frac{\langle 46\rangle\langle 47\rangle^{3}[13][23]^{2}}{s_{123}[12]\langle 45\rangle\langle 56\rangle\langle 67\rangle\langle 4|3+2| 1]\langle 7|1+2| 3]} \\
& -\frac{\langle 24\rangle\langle 14\rangle^{3}[57][56]^{2}}{s_{567}\langle 12\rangle\langle 23\rangle\langle 34\rangle[67]\langle 4|5+6| 7]\langle 1|7+6| 5]} \\
& -\frac{\langle 24\rangle\langle 71\rangle^{2}\langle 6|7+1| 5]\langle 4|3+2| 5]^{3}}{s_{671} s_{234}\langle 23\rangle\langle 34\rangle\langle 67\rangle\langle 1|7+6| 5]\langle 2|3+4| 5]\langle 6|(7+1)(2+3)| 4\rangle} \\
& -\frac{\langle 26\rangle\langle 71\rangle^{2}[35]^{4}}{s_{345}\langle 67\rangle\langle 12\rangle[34][45]\langle 2|3+4| 5]\langle 6|5+4| 3]} .
\end{aligned}
$$


Picking $j=4, l=5, A_{13}$ is given by

$$
\begin{aligned}
A_{13} & =A_{7}^{(0)}\left(1_{q}^{-}, 2_{\bar{q}}^{+}, 3^{+}, 4^{+}, 5_{p}^{+}, 6_{\bar{p}}^{-}, 7^{-}\right)= \\
& -\frac{\left(s_{345}\right)^{2}\langle 27\rangle\langle 17\rangle^{2}\langle 5|4+3| 6]}{s_{712}\langle 12\rangle\langle 34\rangle\langle 45\rangle\langle 2|1+7| 6]\langle 3|4+5| 6]\langle 7|(1+2)(3+4)| 5\rangle} \\
& +\frac{\langle 57\rangle\langle 67\rangle^{2}\langle 5|4+3| 1]\langle 5|4+3| 2]^{2}}{[12]\langle 34\rangle\langle 45\rangle\langle 56\rangle\langle 5|6+7| 1]\langle 7|(1+2)(3+4)| 5\rangle\langle 5|(6+7)(1+2)| 3\rangle} \\
& -\frac{s_{671}^{2}\langle 25\rangle[16]}{\langle 23\rangle\langle 34\rangle\langle 45\rangle[67][71]\langle 2|1+7| 6]\langle 5|6+7| 1]} \\
& +\frac{\langle 27\rangle\langle 71\rangle^{2}[46][45]^{2}}{s_{456}\langle 12\rangle\langle 23\rangle[56]\langle 7|6+5| 4]\langle 3|4+5| 6]} \\
& -\frac{\langle 57\rangle\langle 67\rangle^{2}\langle 2|1+3| 4]\langle 1|2+3| 4]^{2}}{s_{567} s_{123}\langle 12\rangle\langle 23\rangle\langle 56\rangle\langle 7|6+5| 4]\langle 5|(6+7)(1+2)| 3\rangle} .
\end{aligned}
$$

Finally, $A_{14}$ has been computed by selecting $j=1, l=2$

$$
\begin{aligned}
A_{14} & =A_{7}^{(0)}\left(1_{q}^{-}, 2_{\bar{q}}^{+}, 3^{+}, 4^{-}, 5_{p}^{+}, 6_{\bar{p}}^{-}, 7^{+}\right)= \\
& -\frac{\langle 46\rangle^{3}\langle 2|1+7| 3]\langle 1|7+2| 3]^{3}}{s_{456} s_{712}\langle 56\rangle\langle 71\rangle\langle 12\rangle\langle 6|5+4| 3]\langle 7|1+2| 3]\langle 4|(5+6)(7+1)| 2\rangle} \\
& -\frac{[13][23]^{2}\langle 46\rangle^{3}}{s_{123}[12]\langle 56\rangle\langle 67\rangle\langle 4|3+2| 1]\langle 7|1+2| 3]} \\
& +\frac{\langle 24\rangle\langle 46\rangle^{3}\langle 4|3+2| 7]^{3}}{\langle 23\rangle\langle 34\rangle\langle 56\rangle\langle 4|3+2| 1]\langle 4|5+6| 7]\langle 6|(7+1)(2+3)| 4\rangle\langle 4|(5+6)(7+1)| 2\rangle} \\
& -\frac{\langle 24\rangle\langle 14\rangle^{3}[57]^{3}}{s_{567}\langle 12\rangle\langle 23\rangle\langle 34\rangle[56]\langle 1|7+6| 5]\langle 4|5+6| 7]} \\
& -\frac{\langle 24\rangle\langle 16\rangle^{3}\langle 4|3+2| 5]^{3}}{s_{234}\langle 23\rangle\langle 34\rangle\langle 67\rangle\langle 71\rangle\langle 2|3+4| 5]\langle 1|7+6| 5]\langle 6|(7+1)(2+3)| 4\rangle} \\
& -\frac{\langle 16\rangle^{3}[35]^{3}\langle 2|5+4| 3]}{s_{345}[34][45]\langle 67\rangle\langle 71\rangle\langle 12\rangle\langle 6|5+4| 3]\langle 2|3+4| 5]} .
\end{aligned}
$$

\section{$3.2 \quad 4 q+3 g$ sub-leading amplitudes}

We anticipate that the sub-leading amplitudes have fewer and simpler terms than the leading ones,mainly due to the fact that the ordering of the quarks causes the vanishing of many BCFW sub-amplitudes.

The calculation of $B_{1}$ was performed by taking $j=5, l=6$

$$
\begin{aligned}
B_{1} & =A_{7}^{(0)}\left(1_{q}^{+}, 2_{\bar{p}}^{-}, 3^{+}, 4^{+}, 5^{-}, 6_{p}^{+}, 7_{\bar{q}}^{-}\right)= \\
& +\frac{[16]^{2}\langle 25\rangle^{3}}{s_{671}\langle 23\rangle\langle 34\rangle\langle 45\rangle[71]\langle 2|1+7| 6]} \\
& +\frac{\langle 27\rangle^{2}\langle 5|4+3| 6]^{3}}{s_{712} s_{345}\langle 34\rangle\langle 45\rangle\langle 71\rangle\langle 3|4+5| 6]\langle 2|1+7| 6]} \\
& -\frac{\langle 27\rangle^{2}[46]^{3}}{s_{456}\langle 23\rangle[45][56]\langle 71\rangle\langle 3|4+5| 6]} .
\end{aligned}
$$


$B_{2}$ was computed by selecting $j=2, l=3$. The final result is

$$
\begin{aligned}
B_{2} & =A_{7}^{(0)}\left(1_{q}^{+}, 2_{\bar{p}}^{-}, 3^{+}, 4^{-}, 5^{+}, 6_{p}^{+}, 7_{\bar{q}}^{-}\right)= \\
& +\frac{\langle 46\rangle\langle 24\rangle^{3}\langle 4|5+6| 1]^{2}}{\langle 23\rangle\langle 34\rangle\langle 45\rangle\langle 56\rangle[71]\langle 6|(7+1)(2+3)| 4\rangle\langle 4|(5+6)(7+1)| 2\rangle} \\
& +\frac{\langle 46\rangle\langle 27\rangle^{2}\langle 4|5+6| 3]^{3}}{s_{456} s_{712}\langle 45\rangle\langle 56\rangle\langle 71\rangle\langle 6|5+4| 3]\langle 4|(5+6)(7+1)| 2\rangle} \\
& +\frac{\langle 24\rangle^{3}\langle 4|3+2| 5]\langle 7|1+6| 5]^{2}}{s_{671} s_{234}\langle 23\rangle\langle 34\rangle\langle 71\rangle\langle 2|3+4| 5]\langle 6|(7+1)(2+3)| 4\rangle} \\
& -\frac{\langle 27\rangle^{2}[35]^{4}}{s_{345}[34][45]\langle 71\rangle\langle 2|3+4| 5]\langle 6|5+4| 3]} .
\end{aligned}
$$

$B_{3}$ has been obtained by choosing $j=3, l=4$

$$
\begin{aligned}
B_{3} & =A_{7}^{(0)}\left(1_{q}^{+}, 2_{\bar{p}}^{-}, 3^{-}, 4^{+}, 5^{+}, 6_{p}^{+}, 7_{\bar{q}}^{-}\right)= \\
& +\frac{\langle 36\rangle\langle 3|7+2| 1]^{2}}{s_{712}\langle 34\rangle\langle 45\rangle\langle 56\rangle[71]\langle 6|7+1| 2]} \\
& +\frac{\langle 3|4+5| 2]\langle 3|(4+5)(6+1)| 7\rangle^{2}}{s_{345} s_{671}\langle 34\rangle\langle 45\rangle\langle 71\rangle\langle 5|4+3| 2]\langle 6|7+1| 2]} \\
& -\frac{[24]\langle 7|2+3| 4]^{2}}{s_{234}[23][34]\langle 56\rangle\langle 71\rangle\langle 5|4+3| 2]} .
\end{aligned}
$$

The calculation of $B_{4}$ has been performed by selecting $j=3, l=4$

$$
\begin{aligned}
B_{4} & =A_{7}^{(0)}\left(1_{q}^{+}, 2_{\bar{p}}^{+}, 3^{+}, 4^{+}, 5^{-}, 6_{p}^{-}, 7_{\bar{q}}^{-}\right)= \\
& +\frac{\langle 25\rangle\langle 5|6+7| 1]^{2}}{s_{671}\langle 23\rangle\langle 34\rangle\langle 45\rangle[71]\langle 2|1+7| 6]} \\
& +\frac{\langle 5|4+3| 6]\langle 7|(1+2)(3+4)| 5\rangle^{2}}{s_{712} s_{345}\langle 34\rangle\langle 45\rangle\langle 71\rangle\langle 3|4+5| 6]\langle 2|1+7| 6]} \\
& -\frac{[46]\langle 7|6+5| 4]^{2}}{s_{456}\langle 23\rangle[45][56]\langle 71\rangle\langle 3|4+5| 6]} .
\end{aligned}
$$

Setting $j=4, l=5$, we have computed $B_{5}$, arriving at the following expression

$$
\begin{aligned}
B_{5} & =A_{7}^{(0)}\left(1_{q}^{+}, 2_{\bar{p}}^{+}, 3^{+}, 4^{-}, 5^{+}, 6_{p}^{-}, 7_{\bar{q}}^{-}\right)= \\
& +\frac{\langle 24\rangle\langle 46\rangle^{3}\langle 4|3+2| 1]^{2}}{\langle 23\rangle\langle 34\rangle\langle 45\rangle\langle 56\rangle[71]\langle 4|(5+6)(7+1)| 2\rangle\langle 6|(7+1)(2+3)| 4\rangle} \\
& +\frac{\langle 46\rangle^{3}\langle 4|5+6| 3]\langle 7|1+2| 3]^{2}}{s_{456} s_{712}\langle 45\rangle\langle 56\rangle\langle 71\rangle\langle 6|5+4| 3]\langle 4|(5+6)(7+1)| 2\rangle} \\
& +\frac{\langle 24\rangle\langle 67\rangle^{2}\langle 4|3+2| 5]^{3}}{s_{671} s_{234}\langle 23\rangle\langle 34\rangle\langle 71\rangle\langle 2|3+4| 5]\langle 6|(7+1)(2+3)| 4\rangle} \\
& -\frac{\langle 67\rangle^{2}[35]^{4}}{s_{345}[34][45]\langle 71\rangle\langle 2|3+4| 5]\langle 6|5+4| 3]}
\end{aligned}
$$

We have calculated $B_{6}$ by choosing $j=3, l=4$

$$
\begin{aligned}
B_{6} & =A_{7}^{(0)}\left(1_{q}^{+}, 2_{\bar{p}}^{+}, 3^{-}, 4^{+}, 5^{+}, 6_{p}^{-}, 7_{\bar{q}}^{-}\right)= \\
& +\frac{[12]^{2}\langle 36\rangle^{3}}{s_{712}\langle 34\rangle\langle 45\rangle\langle 56\rangle[71]\langle 6|7+1| 2]} \\
& +\frac{\langle 67\rangle^{2}\langle 3|4+5| 2]^{3}}{s_{345} s_{671}\langle 34\rangle\langle 45\rangle\langle 71\rangle\langle 5|4+3| 2]\langle 6|7+1| 2]} \\
& -\frac{\langle 67\rangle^{2}[24]^{3}}{s_{234}[23][34]\langle 56\rangle\langle 71\rangle\langle 5|4+3| 2} .
\end{aligned}
$$


Picking $j=4, l=5, B_{7}$ is simply given by

$$
\begin{aligned}
B_{7} & =A_{7}^{(0)}\left(1_{q}^{+}, 2_{\bar{p}}^{-}, 3^{+}, 4^{+}, 5_{p}^{+}, 6_{\bar{q}}^{-}, 7^{-}\right)= \\
& -\frac{\langle 2|6+7| 1]^{2}}{s_{671}\langle 23\rangle\langle 34\rangle\langle 45\rangle[67][71]} .
\end{aligned}
$$

Selecting $j=4, l=5$, the calculation of $B_{8}$ yields

$$
\begin{aligned}
B_{8} & =A_{7}^{(0)}\left(1_{q}^{+}, 2_{\bar{p}}^{-}, 3^{+}, 4^{-}, 5_{p}^{+}, 6_{\bar{q}}^{-}, 7^{+}\right)= \\
& +\frac{\langle 24\rangle^{3}\langle 6|7+1| 5]^{2}}{s_{671} s_{234}\langle 23\rangle\langle 34\rangle\langle 67\rangle\langle 71\rangle\langle 2|3+4| 5]} \\
& -\frac{\langle 26\rangle^{2}[35]^{3}}{s_{345}[34][45]\langle 67\rangle\langle 71\rangle\langle 2|3+4| 5]} .
\end{aligned}
$$

The last four sub-leading amplitudes $B_{9}-B_{12}$ were obtained by choosing $j=6, l=7$, and read

$$
\begin{aligned}
& B_{9}=A_{7}^{(0)}\left(1_{q}^{+}, 2_{\bar{p}}^{-}, 3^{-}, 4^{+}, 5_{p}^{+}, 6_{\bar{q}}^{-}, 7^{+}\right)= \\
& +\frac{\langle 35\rangle\langle 6|(7+1)(5+4)| 3\rangle^{2}}{s_{345} s_{671}\langle 34\rangle\langle 45\rangle\langle 67\rangle\langle 71\rangle\langle 5|4+3| 2]} \\
& -\frac{[24]\langle 6|2+3| 4]^{2}}{s_{234}[23][34]\langle 67\rangle\langle 71\rangle\langle 5|4+3| 2]}, \\
& B_{10}=A_{7}^{(0)}\left(1_{q}^{+}, 2_{\bar{p}}^{+}, 3^{+}, 4^{+}, 5_{p}^{-}, 6_{\bar{q}}^{-}, 7^{-}\right)= \\
& -\frac{\langle 5|6+7| 1]^{2}}{s_{671}\langle 23\rangle\langle 34\rangle\langle 45\rangle[67][71]} \text {. } \\
& B_{11}=A_{7}^{(0)}\left(1_{q}^{+}, 2_{\bar{p}}^{+}, 3^{+}, 4^{-}, 5_{p}^{-}, 6_{\bar{q}}^{-}, 7^{+}\right)= \\
& +\frac{\langle 24\rangle\langle 6|(7+1)(2+3)| 4\rangle^{2}}{s_{671} s_{234}\langle 23\rangle\langle 34\rangle\langle 67\rangle\langle 71\rangle\langle 2|3+4| 5]} \\
& -\frac{[35]\langle 6|5+4| 3]^{2}}{s_{345}[34][45]\langle 67\rangle\langle 71\rangle\langle 2|3+4| 5]}, \\
& B_{12}=A_{7}^{(0)}\left(1_{q}^{+}, 2_{\bar{p}}^{+}, 3^{-}, 4^{+}, 5_{p}^{-}, 6_{\bar{q}}^{-}, 7^{+}\right)= \\
& +\frac{\langle 35\rangle^{3}\langle 6|7+1| 2]^{2}}{s_{345} s_{671}\langle 34\rangle\langle 45\rangle\langle 67\rangle\langle 71\rangle\langle 5|4+3| 2]} \\
& -\frac{\langle 56\rangle^{2}[24]^{3}}{s_{234}[23][34]\langle 67\rangle\langle 71\rangle\langle 5|4+3| 2]} .
\end{aligned}
$$

\section{$4 \quad$ NMHV six-quark plus one-gluon amplitudes}

Six-quark processes in QCD were first considered in [21]. We discuss here the three different flavor $(p, q$ and $r)$ case. In order to obtain the amplitudes for two or three equal flavors one has to antisymmetrize the expression by adding and substracting amplitudes with the antiquarks exchanged; for more details see [21]. From Eq.(11), one can notice that three different color structure arise: the leading, sub-leading and sub-sub-leading contributions, corresponding to $p=0, p=1$, and $p=2$, respectively. 
For $p=0$, formally the two following possibilities appear : $\alpha_{1}=\bar{i}_{2}, \alpha_{2}=\bar{i}_{3}, \alpha_{3}=\bar{i}_{1}$ and $\alpha_{1}=\bar{i}_{3}, \alpha_{2}=\bar{i}_{1}, \alpha_{3}=$ $\bar{i}_{2}$. The corresponding color factors are

$$
\left(T^{a\left(\sigma_{1}\right)} \ldots T^{a\left(\sigma_{j}\right)}\right)_{i_{1} \bar{i}_{2}}\left(T^{a\left(\sigma_{j+1}\right)} \ldots T^{a\left(\sigma_{l}\right)}\right)_{i_{2} \bar{i}_{3}}\left(T^{a\left(\sigma_{l+1}\right)} \ldots T^{a\left(\sigma_{k}\right)}\right)_{i_{3} \bar{i}_{1}}
$$

and

$$
\left(T^{a\left(\sigma_{1}\right)} \ldots T^{a\left(\sigma_{j}\right)}\right)_{i_{1} \bar{i}_{3}}\left(T^{a\left(\sigma_{j+1}\right)} \ldots T^{a\left(\sigma_{l}\right)}\right)_{i_{2} \bar{i}_{1}}\left(T^{a\left(\sigma_{l+1}\right)} \ldots T^{a\left(\sigma_{k}\right)}\right)_{i_{3} \bar{i}_{2}},
$$

even though the second structure does not contribute to the physical process in interest. To complete the color basis, one has to add also those structures corresponding to the $2 \leftrightarrow 3$ exchange, but those terms do not lead to independent amplitudes.

For $p=1$, the three following color structures have to be taken into account: $\alpha_{1}=\bar{i}_{1}, \alpha_{2}=\bar{i}_{3}, \alpha_{3}=\bar{i}_{2}$, $\alpha_{1}=\bar{i}_{2}, \alpha_{2}=\bar{i}_{1}, \alpha_{3}=\bar{i}_{3}$ and $\alpha_{1}=\bar{i}_{3}, \alpha_{2}=\bar{i}_{2}, \alpha_{3}=\bar{i}_{1}$. It is easy to see that the second structure can be related to the first one by cyclic permutation, and similarly for the third-one. Therefore the only independent sub-leading color form factor corresponds to the string

$$
\frac{-1}{N}\left(T^{a\left(\sigma_{1}\right)} \ldots T^{a\left(\sigma_{j}\right)}\right)_{i_{1} \bar{i}_{1}}\left(T^{a\left(\sigma_{j+1}\right)} \ldots T^{a\left(\sigma_{l}\right)}\right)_{i_{2} \bar{i}_{3}}\left(T^{a\left(\sigma_{l+1}\right)} \ldots T^{a\left(\sigma_{k}\right)}\right)_{i_{3} \bar{i}_{2}}
$$

When $p=2$ things become simpler than in the previous cases, because the only possible configuration is $\alpha \equiv i$. Then the sub-sub-leading color structure is given by

$$
\frac{1}{N^{2}}\left(T^{a\left(\sigma_{1}\right)} \ldots T^{a\left(\sigma_{j}\right)}\right)_{i_{1} \bar{i}_{1}}\left(T^{a\left(\sigma_{j+1}\right)} \ldots T^{a\left(\sigma_{l}\right)}\right)_{i_{2} \bar{i}_{2}}\left(T^{a\left(\sigma_{l+1}\right)} \ldots T^{a\left(\sigma_{k}\right)}\right)_{i_{3} \bar{i}_{3}}
$$

At the level of the partial amplitudes, the string for leading color is written in a generic form as

$$
\left[q\left(g_{\sigma_{1}} \ldots g_{\sigma_{j}}\right) \bar{p}\right]\left[p\left(g_{\sigma_{j+1}} \ldots g_{\sigma_{l}}\right) \bar{r}\right]\left[r\left(g_{\sigma_{l+1}} \ldots g_{\sigma_{l}}\right) \bar{q}\right]
$$

for first leading-color structure and

$$
\left[q\left(g_{\sigma_{1}} \ldots g_{\sigma_{j}}\right) \bar{r}\right]\left[p\left(g_{\sigma_{j+1}} \ldots g_{\sigma_{l}}\right) \bar{q}\right]\left[r\left(g_{\sigma_{l+1}} \ldots g_{\sigma_{l}}\right) \bar{p}\right]
$$

for the second one. For the sub-leading color one has

$$
\left[q\left(g_{\sigma_{1}} \ldots g_{\sigma_{j}}\right) \bar{q}\right]\left[p\left(g_{\sigma_{j+1}} \ldots g_{\sigma_{l}}\right) \bar{r}\right]\left[r\left(g_{\sigma_{l+1}} \ldots g_{\sigma_{l}}\right) \bar{p}\right]
$$

and for the sub-sub-leading case the structure is

$$
\left[q\left(g_{\sigma_{1}} \ldots g_{\sigma_{j}}\right) \bar{q}\right]\left[p\left(g_{\sigma_{j+1}} \ldots g_{\sigma_{l}}\right) \bar{p}\right]\left[r\left(g_{\sigma_{l+1}} \ldots g_{\sigma_{l}}\right) \bar{r}\right]
$$

In order to maintain the same notation along this paper we present the results for the amplitudes using the following order of partons ( $q$ always first)

$$
A\left(q, \bar{q}, g_{1}, \ldots, g_{j}, p, \bar{p}, g_{j+1}, \ldots, g_{k}, r, \bar{r}, g_{k+1}, \ldots g_{l}\right)
$$

or

$$
A\left(q, \bar{p}, g_{1}, \ldots, g_{j}, r, \bar{q}, g_{j+1}, \ldots, g_{k}, p, \bar{r}, g_{k+1}, \ldots, g_{l}\right)
$$

for the leading amplitudes,

$$
A\left(q, \bar{p}, g_{1}, \ldots, g_{j}, p, \bar{q}, g_{j+1}, \ldots, g_{k}, r, \bar{r}, g_{k+1}, \ldots, g_{l}\right)
$$

for the sub-leading ones and

$$
A\left(q, \bar{p}, g_{1}, \ldots, g_{j}, p, \bar{r}, g_{j+1}, \ldots, g_{k}, r, \bar{q}, g_{k+1}, \ldots, g_{l}\right)
$$

for the sub-sub-leading case.

For the sake of presentation, we denote the leading contributions as "A" amplitudes, the sub-leading as "B" and the sub-sub-leading "C". We have found that the number of independent amplitudes is in principle twenty-four: eight for the leading color $\left(A_{1}, \ldots, A_{8}\right)$, twelve for the sub-leading $\left(B_{1}, \ldots, B_{12}\right)$ and four for the sub-sub-leading $\left(C_{1}, \ldots, C_{4}\right)$. 


\subsection{Leading amplitudes}

In order to obtain a leading amplitude the only available gluon should sit in between two fermions of different flavor. It is therefore equivalent, just by flavor relabelling, to insert the gluon in any possible place compatible with the color structure. We have chosen the gluon to be the third particle. Besides, as there exist already three fermions with negative polarization, the gluon should necessarily have positive helicity to build an NMHV amplitude. Those coming from the first color structure are

$$
\begin{array}{ll}
A_{1}=A_{7}^{(0)}\left(1_{q}^{+}, 2_{\bar{q}}^{-}, 3^{+}, 4_{p}^{+}, 5_{\bar{p}}^{-}, 6_{r}^{+}, 7_{\bar{r}}^{-}\right) & A_{3}=A_{7}^{(0)}\left(1_{q}^{+}, 2_{\bar{q}}^{-}, 3^{+}, 4_{p}^{-}, 5_{\bar{p}}^{+}, 6_{r}^{-}, 7_{\bar{r}}^{+}\right) \\
A_{2}=A_{7}^{(0)}\left(1_{q}^{+}, 2_{\bar{q}}^{-}, 3^{+}, 4_{p}^{+}, 5_{\bar{p}}^{-}, 6_{r}^{-}, 7_{\bar{r}}^{+}\right) & A_{4}=A_{7}^{(0)}\left(1_{q}^{-}, 2_{\bar{q}}^{+}, 3^{+}, 4_{p}^{+}, 5_{\bar{p}}^{-}, 6_{r}^{-}, 7_{\bar{r}}^{+}\right),
\end{array}
$$

while those from the second color string

$$
\begin{array}{ll}
A_{5}=A_{7}^{(0)}\left(1_{q}^{+}, 2_{\bar{p}}^{-}, 3^{+}, 4_{r}^{+}, 5_{\bar{q}}^{-}, 6_{p}^{+}, 7_{\bar{r}}^{-}\right) & A_{7}=A_{7}^{(0)}\left(1_{q}^{+}, 2_{\bar{p}}^{+}, 3^{+}, 4_{r}^{+}, 5_{\bar{q}}^{-}, 6_{p}^{-}, 7_{\bar{r}}^{-}\right) \\
A_{6}=A_{7}^{(0)}\left(1_{q}^{+}, 2_{\bar{p}}^{-}, 3^{+}, 4_{r}^{-}, 5_{\bar{q}}^{-}, 6_{p}^{+}, 7_{\bar{r}}^{+}\right) & A_{8}=A_{7}^{(0)}\left(1_{q}^{+}, 2_{\bar{p}}^{+}, 3^{+}, 4_{r}^{-}, 5_{\bar{q}}^{-}, 6_{p}^{-}, 7_{\bar{r}}^{+}\right)
\end{array}
$$

are all vanishing. Below we present the most compact expressions for the four leading amplitudes.

$A_{1}$ has been obtained by picking $j=5, l=6$

$$
\begin{aligned}
A_{1} & =A_{7}^{(0)}\left(1_{q}^{+}, 2_{\bar{q}}^{-}, 3^{+}, 4_{p}^{+}, 5_{\bar{p}}^{-}, 6_{r}^{+}, 7_{\bar{r}}^{-}\right)= \\
& -\frac{\langle 57\rangle^{2}[34]^{2}\langle 27\rangle^{3}\langle 7|6+5| 3]}{\langle 12\rangle\langle 67\rangle\langle 7|1+2| 3]\langle 7|6+5| 4]\langle 2|(3+4)(5+6)| 7\rangle\langle 7|(1+2)(3+4)| 5\rangle} \\
& -\frac{\langle 47\rangle\langle 57\rangle^{2}[13]^{3}}{s_{123}[12]\langle 45\rangle\langle 67\rangle\langle 4|3+2| 1]\langle 7|1+2| 3]} \\
& +\frac{\langle 24\rangle\langle 57\rangle^{2}\langle 7|6+5| 1]\langle 2|3+4| 1]^{2}}{s_{567}\langle 23\rangle\langle 34\rangle\langle 67\rangle\langle 5|6+7| 1]\langle 4|3+2| 1]\langle 2|(3+4)(5+6)| 7\rangle} \\
& -\frac{\langle 24\rangle\langle 25\rangle^{2}[16]^{2}\langle 5|1+7| 6]}{s_{671}\langle 23\rangle\langle 34\rangle\langle 45\rangle[67]\langle 2|1+7| 6]\langle 5|6+7| 1]} \\
& -\frac{\langle 27\rangle^{2}\langle 4|3+5| 6]\langle 5|4+3| 6]^{2}\langle 2|(1+7)(3+4)| 5\rangle}{s_{712} s_{345}\langle 12\rangle\langle 34\rangle\langle 45\rangle\langle 3|4+5| 6]\langle 2|1+7| 6]\langle 7|(1+2)(3+4)| 5\rangle} \\
- & \frac{\langle 27\rangle^{2}[46]^{2}\langle 2|6+5| 4]}{s_{456}\langle 12\rangle\langle 23\rangle[45]\langle 3|4+5| 6]\langle 7|6+5| 4]} .
\end{aligned}
$$

Selecting $j=2, l=3, A_{2}$ yields

$$
\begin{aligned}
A_{2} & =A_{7}^{(0)}\left(1_{q}^{+}, 2_{\bar{q}}^{-}, 3^{+}, 4_{p}^{+}, 5_{\bar{p}}^{-}, 6_{r}^{-}, 7_{\bar{r}}^{+}\right)= \\
& -\frac{\langle 24\rangle\langle 25\rangle^{2}[71]^{2}\langle 5|1+7| 6]}{s_{671}\langle 23\rangle\langle 34\rangle\langle 45\rangle[67]\langle 5|6+7| 1]\langle 2|1+7| 6]} \\
& +\frac{\langle 24\rangle\langle 2|(3+4)(5+6)| 2\rangle^{3}}{\langle 12\rangle\langle 23\rangle\langle 34\rangle\langle 2|3+4| 5]\langle 2|1+7| 6]\langle 4|(5+6)(7+1)| 2\rangle\langle 2|(3+4)(5+6)| 7\rangle} \\
& +\frac{\langle 24\rangle\langle 56\rangle^{2}\langle 7|6+5| 1]\langle 2|3+4| 1]^{2}}{s_{567}\langle 23\rangle\langle 34\rangle\langle 67\rangle\langle 4|3+2| 1]\langle 5|6+7| 1]\langle 2|(3+4)(5+6)| 7\rangle} \\
& +\frac{[35][34]^{2}\langle 26\rangle^{3}}{s_{345}\langle 67\rangle\langle 12\rangle[45]\langle 6|5+4| 3]\langle 2|3+4| 5]} \\
& -\frac{\langle 56\rangle^{2}\langle 4|5+6| 3]\langle 2|1+7| 3]^{3}}{s_{456} s_{712}\langle 12\rangle\langle 45\rangle\langle 7|1+2| 3]\langle 6|5+4| 3]\langle 4|(5+6)(7+1)| 2} \\
& -\frac{\langle 47\rangle\langle 56\rangle^{2}[13]^{3}}{s_{123}[12]\langle 45\rangle\langle 67\rangle\langle 7|1+2| 3]\langle 4|3+2| 1]}
\end{aligned}
$$


Picking $j=2, l=3, A_{3}$ is given by

$$
\begin{aligned}
A_{3} & =A_{7}^{(0)}\left(1_{q}^{+}, 2_{\bar{q}}^{-}, 3^{+}, 4_{p}^{-}, 5_{\bar{p}}^{+}, 6_{r}^{-}, 7_{\bar{r}}^{+}\right)= \\
& -\frac{\langle 46\rangle^{2}[71]^{2}\langle 24\rangle^{3}\langle 4|(5+6)(2+3)| 4\rangle}{\langle 23\rangle\langle 34\rangle\langle 45\rangle\langle 4|5+6| 7]\langle 4|3+2| 1]\langle 6|(7+1)(2+3)| 4\rangle\langle 4|(5+6)(7+1)| 2\rangle} \\
& +\frac{\langle 24\rangle^{3}\langle 6|7+1| 5]^{3}}{s_{671}\langle 23\rangle\langle 34\rangle\langle 67\rangle\langle 2|3+4| 5]\langle 1|7+6| 5]\langle 6|(7+1)(2+3)| 4\rangle} \\
& -\frac{[57]^{2}\langle 24\rangle^{3}\langle 1|5+6| 7]}{s_{567}\langle 12\rangle\langle 23\rangle\langle 34\rangle[67]\langle 4|5+6| 7]\langle 1|7+6| 5]} \\
& +\frac{\langle 26\rangle^{3}[35]^{3}}{s_{345}\langle 67\rangle\langle 12\rangle[45]\langle 2|3+4| 5]\langle 6|5+4| 3]} \\
& -\frac{\langle 46\rangle^{2}\langle 4|5+6| 3]\langle 2|1+7| 3]^{3}}{s_{456} s_{712}\langle 12\rangle\langle 45\rangle\langle 7|1+2| 3]\langle 6|5+4| 3]\langle 4|(5+6)(7+1)| 2\rangle} \\
& -\frac{\langle 47\rangle\langle 46\rangle^{2}[13]^{3}}{s_{123}[12]\langle 45\rangle\langle 67\rangle\langle 7|1+2| 3]\langle 4|3+2| 1]} .
\end{aligned}
$$

$A_{4}$ has been computed by choosing $j=1, l=7$, as

$$
\begin{aligned}
A_{4} & =A_{7}^{(0)}\left(1_{q}^{-}, 2_{\bar{q}}^{+}, 3^{+}, 4_{p}^{+}, 5_{\bar{p}}^{-}, 6_{r}^{-}, 7_{\bar{r}}^{+}\right)= \\
& +\frac{\langle 26\rangle\langle 16\rangle^{2}[35][34]^{2}}{s_{345}\langle 67\rangle\langle 12\rangle[45]\langle 6|5+4| 3]\langle 2|3+4| 5]} \\
& +\frac{\langle 46\rangle\langle 56\rangle^{2}\langle 16\rangle^{2}[23]^{2}\langle 6|7+1| 3]}{\langle 45\rangle\langle 67\rangle\langle 6|7+1| 2]\langle 6|5+4| 3]\langle 1|(2+3)(4+5)| 6\rangle\langle 6|(7+1)(2+3)| 4\rangle} \\
& +\frac{\left(s_{234}\right)^{2}\langle 24\rangle\langle 16\rangle^{2}\langle 6|7+1| 5]}{s_{671}\langle 23\rangle\langle 34\rangle\langle 67\rangle\langle 1|7+6| 5]\langle 2|3+4| 5]\langle 6|(7+1)(2+3)| 4\rangle} \\
& -\frac{\langle 24\rangle\langle 1|5+6| 7]^{3}}{s_{567}\langle 12\rangle\langle 23\rangle\langle 34\rangle[67]\langle 4|5+6| 7]\langle 1|7+6| 5]} \\
& -\frac{\langle 56\rangle^{2}\langle 2|3+1| 7]\langle 1|2+3| 7]^{2}\langle 1|(2+3)(6+5)| 4\rangle}{s_{123} s_{456}\langle 12\rangle\langle 23\rangle\langle 45\rangle\langle 3|2+1| 7]\langle 4|5+6| 7]\langle 1|(2+3)(4+5)| 6\rangle} \\
- & \frac{\langle 56\rangle^{2}[72]^{2}\langle 4|1+7| 2]}{s_{712}[12]\langle 34\rangle\langle 45\rangle\langle 6|7+1| 2]\langle 3|2+1| 7]} .
\end{aligned}
$$

\section{2 $\quad$ Sub-leading amplitudes}

At this level, the gluon may be at the third, fifth or seventh place. It is easy to show that two inequivalent choices for NMHV amplitudes are $3^{+}$and $5^{+}$. If the gluon is the third particle, one has the following amplitudes

$$
\begin{array}{ll}
B_{1}=A_{7}^{(0)}\left(1_{q}^{+}, 2_{\bar{p}}^{-}, 3^{+}, 4_{p}^{+}, 5_{\bar{q}}^{-}, 6_{r}^{+}, 7_{\bar{r}}^{-}\right) & B_{3}=A_{7}^{(0)}\left(1_{q}^{+}, 2_{\bar{p}}^{+}, 3^{+}, 4_{p}^{-}, 5_{\bar{q}}^{-}, 6_{r}^{+}, 7_{\bar{r}}^{-}\right) \\
B_{2}=A_{7}^{(0)}\left(1_{q}^{+}, 2_{\bar{p}}^{-}, 3^{+}, 4_{p}^{+}, 5_{\bar{q}}^{-}, 6_{r}^{-}, 7_{\bar{r}}^{+}\right) & B_{4}=A_{7}^{(0)}\left(1_{q}^{+}, 2_{\bar{p}}^{+}, 3^{+}, 4_{p}^{-}, 5_{\bar{q}}^{-}, 6_{r}^{-}, 7_{\bar{r}}^{+}\right),
\end{array}
$$

while if the gluon is $5^{+}$one has to consider

$$
\begin{array}{ll}
B_{5}=A_{7}^{(0)}\left(1_{q}^{+}, 2_{\bar{p}}^{-}, 3_{p}^{+}, 4_{\bar{q}}^{-}, 5^{+}, 6_{r}^{+}, 7_{\bar{r}}^{-}\right) & B_{9}=A_{7}^{(0)}\left(1_{q}^{-}, 2_{\bar{p}}^{-}, 3_{p}^{+}, 4_{\bar{q}}^{+}, 5^{+}, 6_{r}^{+}, 7_{\bar{r}}^{-}\right) \\
B_{6}=A_{7}^{(0)}\left(1_{q}^{+}, 2_{\bar{p}}^{-}, 3_{p}^{+}, 4_{\bar{q}}^{-}, 5^{+}, 6_{r}^{-}, 7_{\bar{r}}^{+}\right) & B_{10}=A_{7}^{(0)}\left(1_{q}^{-}, 2_{\bar{p}}^{-}, 3_{p}^{+}, 4_{\bar{q}}^{+}, 5^{+}, 6_{r}^{-}, 7_{\bar{r}}^{+}\right) \\
B_{7}=A_{7}^{(0)}\left(1_{q}^{+}, 2_{\bar{p}}^{+}, 3_{p}^{-}, 4_{\bar{q}}^{-}, 5^{+}, 6_{r}^{+}, 7_{\bar{r}}^{-}\right) & B_{11}=A_{7}^{(0)}\left(1_{q}^{-}, 2_{\bar{p}}^{+}, 3_{p}^{-}, 4_{\bar{q}}^{+}, 5^{+}, 6_{r}^{+}, 7_{\bar{r}}^{-}\right) \\
B_{8}=A_{7}^{(0)}\left(1_{q}^{+}, 2_{\bar{p}}^{+}, 3_{p}^{-}, 4_{\bar{q}}^{-}, 5^{+}, 6_{r}^{-}, 7_{\bar{r}}^{+}\right) & B_{12}=A_{7}^{(0)}\left(1_{q}^{-}, 2_{\bar{p}}^{+}, 3_{p}^{-}, 4_{\bar{q}}^{+}, 5^{+}, 6_{r}^{-}, 7_{\bar{r}}^{+}\right) .
\end{array}
$$

Half of those amplitudes can be related to the others by a simple exchange of quarks, corresponding to a "flip" operation. 
By selecting $j=2, l=3$, one has for $B_{1}$ the following expression

$$
\begin{aligned}
B_{1} & =A_{7}^{(0)}\left(1_{q}^{+}, 2_{\bar{p}}^{-}, 3^{+}, 4_{p}^{+}, 5_{\bar{q}}^{-}, 6_{r}^{+}, 7_{\bar{r}}^{-}\right)= \\
& +\frac{\langle 57\rangle^{2}\langle 2|3+4| 1]^{2}}{s_{234} s_{567}\langle 23\rangle\langle 34\rangle\langle 67\rangle\langle 5|6+7| 1]} \\
& +\frac{\langle 25\rangle^{2}[16]^{2}}{s_{671}\langle 23\rangle\langle 34\rangle[67]\langle 5|6+7| 1]},
\end{aligned}
$$

while $B_{2}$ is obtained by flipping

$$
B_{2}=A_{7}^{(0)}\left(1_{q}^{+}, 2_{\bar{p}}^{-}, 3^{+}, 4_{p}^{+}, 5_{\bar{q}}^{-}, 6_{r}^{-}, 7_{\bar{r}}^{+}\right)=-B_{1}(\text { flip } 6 \leftrightarrow 7) .
$$

Choosing $j=5, l=6$, the calculation for $B_{3}$ yields

$$
\begin{aligned}
B_{3} & =A_{7}^{(0)}\left(1_{q}^{+}, 2_{\bar{p}}^{+}, 3^{+}, 4_{p}^{-}, 5_{\bar{q}}^{-}, 6_{r}^{+}, 7_{\bar{r}}^{-}\right)= \\
& +\frac{\langle 57\rangle^{2}\langle 4|3+2| 1]^{2}}{s_{234} s_{567}\langle 23\rangle\langle 34\rangle\langle 67\rangle\langle 5|6+7| 1]} \\
& +\frac{\langle 45\rangle^{2}[16]^{2}}{s_{671}\langle 23\rangle\langle 34\rangle[67]\langle 5|6+7| 1]},
\end{aligned}
$$

and $B_{4}$ follows by performing the same flip as before

$$
B_{4}=A_{7}^{(0)}\left(1_{q}^{+}, 2_{\bar{p}}^{+}, 3^{+}, 4_{p}^{-}, 5_{\bar{q}}^{-}, 6_{r}^{-}, 7_{\bar{r}}^{+}\right)=-B_{3}(\text { flip }, 6 \leftrightarrow 7) .
$$

$B_{5}$ has been computed by selecting $j=7, l=1$,

$$
\begin{aligned}
B_{5} & =A_{7}^{(0)}\left(1_{q}^{+}, 2_{\bar{p}}^{-}, 3_{p}^{+}, 4_{\bar{q}}^{-}, 5^{+}, 6_{r}^{+}, 7_{\bar{r}}^{-}\right)= \\
& +\frac{\langle 46\rangle\langle 47\rangle^{2}[13]^{2}}{s_{123}[23]\langle 45\rangle\langle 56\rangle\langle 67\rangle\langle 4|3+2| 1]} \\
& +\frac{\langle 24\rangle^{2}\langle 6|5+7| 1]\langle 7|6+5| 1]^{2}}{s_{234} s_{567}\langle 23\rangle\langle 56\rangle\langle 67\rangle\langle 4|3+2| 1]\langle 5|6+7| 1]} \\
& +\frac{\langle 24\rangle^{2}[16]^{2}}{s_{671}\langle 23\rangle\langle 45\rangle[67]\langle 5|6+7| 1]},
\end{aligned}
$$

and $B_{6}$ by taking $j=4, l=5$,

$$
\begin{aligned}
B_{6}= & A_{7}^{(0)}\left(1_{q}^{+}, 2_{\bar{p}}^{-}, 3_{p}^{+}, 4_{\bar{q}}^{-}, 5^{+}, 6_{r}^{-}, 7_{\bar{r}}^{+}\right)= \\
& -\frac{[71]^{2}\langle 24\rangle^{2}\langle 46\rangle^{3}}{\langle 23\rangle\langle 45\rangle\langle 56\rangle\langle 4|3+2| 1]\langle 4|5+6| 7]\langle 6|(7+1)(2+3)| 4\rangle} \\
& +\frac{[13]^{2}\langle 46\rangle^{3}}{s_{123}[23]\langle 45\rangle\langle 56\rangle\langle 67\rangle\langle 4|3+2| 1]} \\
& +\frac{\langle 24\rangle^{2}[57]^{3}}{s_{567}\langle 23\rangle[67]\langle 1|7+6| 5]\langle 4|5+6| 7]} \\
& +\frac{\langle 24\rangle^{2}\langle 6|7+1| 5]^{3}}{s_{671} s_{234}\langle 23\rangle\langle 67\rangle\langle 1|7+6| 5]\langle 6|(7+1)(2+3)| 4\rangle} .
\end{aligned}
$$

$B_{7}$ can be obtained from $B_{5}$ by a flip operation,

$$
B_{7}=A_{7}^{(0)}\left(1_{q}^{+}, 2_{\bar{p}}^{+}, 3_{p}^{-}, 4_{\bar{q}}^{-}, 5^{+}, 6_{r}^{+}, 7_{\bar{r}}^{-}\right)=-B_{5}(\text { flip }, 2 \leftrightarrow 3),
$$

as can $B_{8}$ from $B_{6}$,

$$
B_{8}=A_{7}^{(0)}\left(1_{q}^{+}, 2_{\bar{p}}^{+}, 3_{p}^{-}, 4_{\bar{q}}^{-}, 5^{+}, 6_{r}^{-}, 7_{\bar{r}}^{+}\right)=-B_{6}(\text { flip } 2 \leftrightarrow 3) .
$$


We have computed $B_{9}$ by selecting $j=1, l=3$,

$$
\begin{aligned}
B_{9} & =A_{7}^{(0)}\left(1_{q}^{-}, 2_{\bar{p}}^{-}, 3_{p}^{+}, 4_{\bar{q}}^{+}, 5^{+}, 6_{r}^{+}, 7_{\bar{r}}^{-}\right)= \\
& +\frac{\langle 12\rangle^{2}\langle 2|4+5| 6]^{2}}{\langle 23\rangle\langle 45\rangle[67]\langle 5|(6+7)(1+3)| 2\rangle\langle 2|(4+5)(6+7)| 1\rangle} \\
& +\frac{\langle 12\rangle^{2}\langle 7|6+5| 4]^{2}\langle 6|(5+7)(1+3)| 2\rangle}{s_{567} s_{123}\langle 23\rangle\langle 56\rangle\langle 67\rangle\langle 1|2+3| 4]\langle 5|(6+7)(1+3)| 2\rangle} \\
& +\frac{\langle 17\rangle^{2}[34]^{2}\langle 6|2+4| 3]}{s_{234}[23]\langle 56\rangle\langle 67\rangle\langle 5|4+2| 3]\langle 1|2+3| 4]} \\
& +\frac{\langle 17\rangle^{2}\langle 2|5+4| 3]^{2}}{s_{671}\langle 45\rangle\langle 67\rangle\langle 5|4+2| 3]\langle 2|(4+5)(6+7)| 1\rangle}
\end{aligned}
$$

The calculation of $B_{10}$ has been performed by choosing $j=2, l=3$,

$$
\begin{aligned}
B_{10} & =A_{7}^{(0)}\left(1_{q}^{-}, 2_{\bar{p}}^{-}, 3_{p}^{+}, 4_{\bar{q}}^{+}, 5^{+}, 6_{r}^{-}, 7_{\bar{r}}^{+}\right)= \\
& +\frac{\langle 46\rangle\langle 16\rangle^{2}\langle 6|5+4| 3]^{2}}{[23]\langle 45\rangle\langle 56\rangle\langle 67\rangle\langle 1|(2+3)(4+5)| 6\rangle\langle 6|(7+1)(2+3)| 4\rangle} \\
& +\frac{\langle 16\rangle^{2}\langle 6|7+1| 5]\langle 2|3+4| 5]^{2}}{s_{671} s_{234}\langle 23\rangle\langle 67\rangle\langle 1|7+6| 5]\langle 6|(7+1)(2+3)| 4\rangle} \\
& +\frac{\langle 12\rangle^{2}[57]^{3}}{s_{567}\langle 23\rangle[67]\langle 4|5+6| 7]\langle 1|7+6| 5]} \\
& -\frac{\langle 46\rangle\langle 12\rangle^{2}\langle 6|5+4| 7]^{2}}{s_{123}\langle 23\rangle\langle 45\rangle\langle 56\rangle\langle 4|5+6| 7]\langle 1|(2+3)(4+5)| 6\rangle} .
\end{aligned}
$$

Finally, a flip operation relates $B_{11}$ to $B_{9}$,

$$
B_{11}=A_{7}^{(0)}\left(1_{\bar{q}}^{-}, 2_{\bar{p}}^{+}, 3_{p}^{-}, 4_{\bar{q}}^{+}, 5^{+}, 6_{r}^{+}, 7_{\bar{r}}^{-}\right)=-B_{9}(\text { flip } 2 \leftrightarrow 3),
$$

and $B_{12}$ to $B_{10}$

$$
B_{12}=A_{7}^{(0)}\left(1_{q}^{-}, 2_{\bar{p}}^{+}, 3_{p}^{-}, 4_{\bar{q}}^{+}, 5^{+}, 6_{r}^{-}, 7_{\bar{r}}^{+}\right)=-B_{10}(\text { flip } 2 \leftrightarrow 3)
$$

\subsection{Sub-sub-leading amplitudes}

Due to the flavor configuration of the sub-sub-leading amplitudes, it is equivalent to insert the gluon in the middle of any quark-antiquark pair. The independent amplitudes we have computed are

$$
\begin{array}{ll}
C_{1}=A_{7}^{(0)}\left(1_{q}^{+}, 2_{\bar{p}}^{-}, 3^{+}, 4_{p}^{+}, 5_{\bar{r}}^{-}, 6_{r}^{+}, 7_{\bar{q}}^{-}\right) & C_{3}=A_{7}^{(0)}\left(1_{q}^{+}, 2_{\bar{p}}^{+}, 3^{+}, 4_{p}^{-}, 5_{\bar{r}}^{-}, 6_{r}^{+}, 7_{\bar{q}}^{-}\right) \\
C_{2}=A_{7}^{(0)}\left(1_{q}^{+}, 2_{\bar{p}}^{-}, 3^{+}, 4_{p}^{+}, 5_{\bar{r}}^{+}, 6_{r}^{-}, 7_{\bar{q}}^{-}\right) & C_{4}=A_{7}^{(0)}\left(1_{q}^{-}, 2_{\bar{p}}^{-}, 3^{+}, 4_{p}^{+}, 5_{\bar{r}}^{-}, 6_{r}^{+}, 7_{\bar{q}}^{+}\right) .
\end{array}
$$

We were able to compute $C_{1}$, by picking $j=2, l=3$, as

$$
\begin{aligned}
C_{1} & =A_{7}^{(0)}\left(1_{q}^{+}, 2_{\bar{p}}^{-}, 3^{+}, 4_{p}^{+}, 5_{\bar{r}}^{-}, 6_{r}^{+}, 7_{\bar{q}}^{-}\right)= \\
& -\frac{\langle 25\rangle^{2}[61]^{2}\langle 2|6+7| 1]}{s_{671}\langle 23\rangle\langle 34\rangle[71]\langle 2|1+7| 6]\langle 5|6+7| 1]} \\
& -\frac{\langle 57\rangle^{2}\langle 2|3+4| 1]^{2}\langle 2|(3+4)(6+7)| 5\rangle}{s_{234} s_{567}\langle 23\rangle\langle 34\rangle\langle 56\rangle\langle 5|6+7| 1]\langle 2|(3+4)(5+6)| 7\rangle} \\
& -\frac{\langle 27\rangle^{3}\langle 4|3+2| 6]\langle 2|3+4| 6]^{2}}{\langle 71\rangle\langle 23\rangle\langle 34\rangle[56]\langle 2|1+7| 6]\langle 4|(5+6)(7+1)| 2\rangle\langle 2|(3+4)(5+6)| 7\rangle} \\
& -\frac{\langle 24\rangle\langle 27\rangle^{2}[16]\langle 2|3+4| 6]^{2}}{\langle 23\rangle\langle 34\rangle[56]\langle 2|1+7| 6]\langle 4|(5+6)(7+1)| 2\rangle\langle 2|(3+4)(5+6)| 7\rangle} \\
& -\frac{\langle 27\rangle^{2}\langle 5|6+4| 3]^{2}}{s_{456} s_{712}\langle 56\rangle\langle 71\rangle\langle 4|(5+6)(7+1)| 2\rangle} .
\end{aligned}
$$


Using the same tag, the amplitude $C_{2}$ is given by

$$
\begin{aligned}
C_{2} & =A_{7}^{(0)}\left(1_{q}^{+}, 2_{\bar{p}}^{-}, 3^{+}, 4_{p}^{+}, 5_{\bar{r}}^{+}, 6_{r}^{-}, 7_{\bar{q}}^{-}\right)= \\
& -\frac{\langle 27\rangle^{3}\langle 4|3+2| 6]\langle 2|3+4| 5]^{2}}{\langle 71\rangle\langle 23\rangle\langle 34\rangle[56]\langle 2|1+7| 6]\langle 4|(5+6)(7+1)| 2\rangle\langle 2|(3+4)(5+6)| 7\rangle} \\
& -\frac{\langle 24\rangle\langle 27\rangle^{2}[16]\langle 2|3+4| 5]^{2}}{\langle 23\rangle\langle 34\rangle[56]\langle 2|1+7| 6]\langle 4|(5+6)(7+1)| 2\rangle\langle 2|(3+4)(5+6)| 7\rangle} \\
& -\frac{\langle 2|6+7| 1]^{3}}{s_{671}\langle 23\rangle\langle 34\rangle[71]\langle 2|1+7| 6]\langle 5|6+7| 1]} \\
& -\frac{\langle 67\rangle^{2}\langle 2|3+4| 1]^{2}\langle 2|(3+4)(6+7)| 5\rangle}{s_{234} s_{567}\langle 23\rangle\langle 34\rangle\langle 56\rangle\langle 5|6+7| 1]\langle 2|(3+4)(5+6)| 7\rangle} \\
& -\frac{\langle 27\rangle^{2}\langle 6|5+4| 3]^{2}}{s_{456} s_{712}\langle 56\rangle\langle 71\rangle\langle 4|(5+6)(7+1)| 2\rangle} .
\end{aligned}
$$

$C_{3}$ was computed by setting $j=1, l=2$, leading to

$$
\begin{aligned}
C_{3} & =A_{7}^{(0)}\left(1_{q}^{+}, 2_{\bar{p}}^{+}, 3^{+}, 4_{p}^{-}, 5_{\bar{r}}^{-}, 6_{r}^{+}, 7_{\bar{q}}^{-}\right)= \\
& -\frac{\langle 57\rangle^{2}\langle 4|3+2| 1]^{2}\langle 2|(3+4)(6+7)| 5\rangle}{s_{234} s_{567}\langle 23\rangle\langle 34\rangle\langle 56\rangle\langle 5|6+7| 1]\langle 2|(3+4)(5+6)| 7\rangle} \\
& -\frac{\langle 57\rangle^{3}\langle 7|1+2| 3]^{2}}{\langle 56\rangle\langle 71\rangle\langle 7|6+5| 4]\langle 2|(3+4)(5+6)| 7\rangle\langle 7|(1+2)(3+4)| 5\rangle} \\
& -\frac{\langle 7|4+5| 6]^{3}}{s_{456}\langle 71\rangle\langle 23\rangle[56]\langle 3|4+5| 6]\langle 7|6+5| 4\rangle} \\
& +\frac{\langle 45\rangle^{2}\langle 7|1+2| 6]^{3}}{s_{712}\langle 34\rangle\langle 71\rangle\langle 2|1+7| 6]\langle 3|4+5| 6]\langle 7|(1+2)(3+4)| 5\rangle} \\
& -\frac{\langle 45\rangle^{2}[16]^{2}\langle 2|6+7| 1]}{s_{671}\langle 23\rangle\langle 34\rangle[71]\langle 5|6+7| 1]\langle 2|1+7| 6]} .
\end{aligned}
$$

Finally, $C_{4}$ was calculated by picking $j=1, l=2$,

$$
\begin{aligned}
C_{4} & =A_{7}^{(0)}\left(1_{q}^{-}, 2_{\bar{p}}^{-}, 3^{+}, 4_{p}^{+}, 5_{\bar{r}}^{-}, 6_{r}^{+}, 7_{\bar{q}}^{+}\right)= \\
& -\frac{\langle 25\rangle^{2}[67]^{2}\langle 2|6+7| 1]}{s_{671}\langle 23\rangle\langle 34\rangle[71]\langle 2|1+7| 6]\langle 5|6+7| 1]} \\
& -\frac{\langle 2|(3+4)(6+7)| 5\rangle^{3}}{s_{234} s_{567}\langle 23\rangle\langle 34\rangle\langle 56\rangle\langle 5|6+7| 1]\langle 2|(3+4)(5+6)| 7\rangle} \\
& -\frac{\langle 27\rangle\langle 12\rangle^{2}\langle 4|3+2| 6]\langle 2|3+4| 6]^{2}}{\langle 71\rangle\langle 23\rangle\langle 34\rangle[56]\langle 2|1+7| 6]\langle 4|(5+6)(7+1)| 2\rangle\langle 2|(3+4)(5+6)| 7\rangle} \\
& -\frac{\langle 24\rangle\langle 12\rangle^{2}[16]\langle 2|3+4| 6]^{2}}{\langle 23\rangle\langle 34\rangle[56]\langle 2|1+7| 6]\langle 4|(5+6)(7+1)| 2\rangle\langle 2|(3+4)(5+6)| 7\rangle} \\
& -\frac{\langle 12\rangle^{2}\langle 5|6+4| 3]^{2}}{s_{456} s_{712}\langle 56\rangle\langle 71\rangle\langle 4|(5+6)(7+1)| 2\rangle} .
\end{aligned}
$$

\section{Conclusions}

In this paper we presented all the four-quark plus three-gluon and six-quark plus one-gluon tree level NMHV helicity amplitudes. They were computed using the BCFW recursion relations. With these results the full set of tree level helicity amplitudes, up to seven partons, becomes available. Our formulae have been tested in all possible collinear and soft limits, which provides a very strict check for the correctness of the amplitudes. $\stackrel{\ddagger}{\ddagger}$.

\footnotetext{
$\ddagger$ Strictly speaking they are correct up to terms that must vanish in all possible soft and collinear limits, which are very unlikely to exist.
} 
These amplitudes are a main ingredient for the calculation of multijets cross-sections in hadronic colliders. As expected, we have obtained very compact expressions, allowing for a more convenient implementation in computer codes than those coming from automatic tree level computational methods.

Acknowledgements. This work has been partially supported by ANPCYT, UBACyT and CONICET.

\section{A Appendix: six-parton NMHV results}

For the sake of completeness, we provide in this appendix the explicit results for the four-quark plus two-gluon and the six-quark NMHV amplitudes.

\section{A.1 NMHV four-quark plus two-gluon amplitudes}

According to the color structure, there are again two different contributions, the leading "A" and the sub-leading "B" amplitudes. The truly independent ones are

$$
\begin{array}{ll}
A_{1}=A_{6}^{(0)}\left(1_{q}^{+}, 2_{\bar{q}}^{-}, 3^{+}, 4^{-}, 5_{p}^{+}, 6_{\bar{p}}^{-}\right) & A_{4}=A_{6}^{(0)}\left(1_{q}^{+}, 2_{\bar{q}}^{-}, 3^{+}, 4_{p}^{+}, 5_{\bar{p}}^{-}, 6^{-}\right) \\
A_{2}=A_{6}^{(0)}\left(1_{q}^{+}, 2_{\bar{q}}^{-}, 3^{-}, 4^{+}, 5_{p}^{+}, 6_{\bar{p}}^{-}\right) & A_{5}=A_{6}^{(0)}\left(1_{q}^{+}, 2_{\bar{q}}^{-}, 3^{+}, 4_{p}^{-}, 5_{\bar{p}}^{+}, 6^{-}\right) \\
A_{3}=A_{6}^{(0)}\left(1_{q}^{+}, 2_{\bar{q}}^{-}, 3^{+}, 4^{-}, 5_{p}^{-}, 6_{\bar{p}}^{+}\right) & A_{6}=A_{6}^{(0)}\left(1_{q}^{+}, 2_{\bar{q}}^{-}, 3^{-}, 4_{p}^{-}, 5_{\bar{p}}^{+}, 6^{+}\right)
\end{array}
$$

for leading, and

$$
\begin{array}{ll}
B_{1}=A_{6}^{(0)}\left(1_{q}^{+}, 2_{\bar{p}}^{-}, 3^{+}, 4^{-}, 5_{p}^{+}, 6_{\bar{q}}^{-}\right) & B_{5}=A_{6}^{(0)}\left(1_{q}^{+}, 2_{\bar{p}}^{-}, 3^{+}, 4_{p}^{+}, 5_{\bar{q}}^{-}, 6^{-}\right) \\
B_{2}=A_{6}^{(0)}\left(1_{q}^{+}, 2_{\bar{p}}^{-}, 3^{-}, 4^{+}, 5_{p}^{+}, 6_{\bar{q}}^{-}\right) & B_{6}=A_{6}^{(0)}\left(1_{q}^{+}, 2_{\bar{p}}^{+}, 3^{+}, 4_{p}^{-}, 5_{\bar{q}}^{-}, 6^{-}\right) \\
B_{3}=A_{6}^{(0)}\left(1_{q}^{+}, 2_{\bar{p}}^{+}, 3^{+}, 4^{-}, 5_{p}^{-}, 6_{\bar{q}}\right) & B_{7}=A_{6}^{(0)}\left(1_{q}^{+}, 2_{\bar{p}}^{+}, 3^{-}, 4_{p}^{-}, 5_{\bar{q}}^{-}, 6^{+}\right) \\
B_{4}=A_{6}^{(0)}\left(1_{q}^{+}, 2_{\bar{p}}^{+}, 3^{-}, 4^{+}, 5_{p}^{-}, 6_{\bar{q}}^{-}\right) &
\end{array}
$$

for the sub-leading color contribution. All the other amplitudes can be obtained by the use of discrete symmetries.

\section{A.1.1 Leading amplitudes}

The most compact results we obtain for the six independent helicity amplitudes are

$$
\begin{aligned}
A_{1} & =A_{6}^{(0)}\left(1_{q}^{+}, 2_{\bar{q}}^{-}, 3^{+}, 4^{-}, 5_{p}^{+}, 6_{\bar{p}}^{-}\right)= \\
& -\frac{\langle 2|1+6| 3]\langle 26\rangle^{2}[35]^{3}}{s_{612}\langle 12\rangle[34][45]\langle 2|1+6| 5]\langle 6|1+2| 3]} \\
& -\frac{[13]^{3}\langle 46\rangle^{3}}{s_{123}[12]\langle 56\rangle\langle 4|2+3| 1]\langle 6|1+2| 3]} \\
& -\frac{\langle 4|6+1| 5][15]^{2}\langle 24\rangle^{3}}{s_{234}\langle 23\rangle\langle 34\rangle[56]\langle 2|6+1| 5]\langle 4|5+6| 1]} \\
A_{2}= & A_{6}^{(0)}\left(1_{q}^{+}, 2_{\bar{q}}^{-}, 3^{-}, 4^{+}, 5_{p}^{+}, 6_{\bar{p}}^{-}\right)= \\
- & \frac{\langle 2|1+6| 4]\langle 26\rangle^{2}[45]^{2}}{s_{612}\langle 12\rangle[34]\langle 2|1+6| 5]\langle 6|1+2| 3]} \\
- & \frac{\langle 5|2+3| 1]\langle 6|1+3| 2]\langle 6|2+3| 1]^{2}}{s_{123}[12][23]\langle 45\rangle\langle 56\rangle\langle 4|2+3| 1]\langle 6|1+2| 3]} \\
- & \frac{\langle 3|6+1| 5][15]^{2}\langle 23\rangle^{2}}{s_{234}\langle 34\rangle[56]\langle 2|6+1| 5]\langle 4|5+6| 1]},
\end{aligned}
$$




$$
\begin{aligned}
& A_{3}=A_{6}^{(0)}\left(1_{q}^{+}, 2_{\bar{q}}^{-}, 3^{+}, 4^{-}, 5_{p}^{-}, 6_{\bar{p}}^{+}\right)= \\
& +\frac{\langle 4|2+3| 5][16]^{2}\langle 24\rangle^{3}}{s_{234}\langle 23\rangle\langle 34\rangle[56]\langle 4|3+2| 1]\langle 2|3+4| 5]} \\
& -\frac{[35]\langle 2|5+4| 3]^{3}}{s_{345}\langle 12\rangle[34][45]\langle 2|3+4| 5]\langle 6|1+2| 3]} \\
& -\frac{\langle 46\rangle\langle 45\rangle^{2}[13]^{3}}{s_{123}[12]\langle 56\rangle\langle 4|3+2| 1]\langle 6|1+2| 3]}, \\
& A_{4}=A_{6}^{(0)}\left(1_{q}^{+}, 2_{\bar{q}}^{-}, 3^{+}, 4_{p}^{+}, 5_{\bar{p}}^{-}, 6^{-}\right)= \\
& +\frac{[35][34]^{2}\langle 26\rangle^{3}}{s_{612}\langle 12\rangle[45]\langle 2|6+1| 5]\langle 6|1+2| 3]} \\
& +\frac{\langle 46\rangle\langle 56\rangle^{2}[13]^{3}}{s_{123}[12]\langle 45\rangle\langle 4|3+2| 1]\langle 6|5+4| 3]} \\
& {[15]\langle 24\rangle\langle 2|5+6| 1]^{2}} \\
& -\overline{\langle 23\rangle\langle 34\rangle[56][61]\langle 2|6+1| 5]\langle 4|5+6| 1]} \text {, } \\
& A_{5}=A_{6}^{(0)}\left(1_{q}^{+}, 2_{\bar{q}}^{-}, 3^{+}, 4_{p}^{-}, 5_{\bar{p}}^{+}, 6^{-}\right)= \\
& -\frac{\langle 24\rangle^{3}[15]^{3}}{\langle 23\rangle\langle 34\rangle[56][61]\langle 4|3+2| 1]\langle 2|3+4| 5]} \\
& +\frac{\langle 26\rangle^{3}[35]^{3}}{s_{345}\langle 12\rangle[45]\langle 2|3+4| 5]\langle 6|5+4| 3]} \\
& -\frac{\langle 46\rangle^{3}[13]^{3}}{s_{123}[12]\langle 45\rangle\langle 6|1+2| 3]\langle 4|3+2| 1]}, \\
& A_{6}=A_{6}^{(0)}\left(1_{q}^{+}, 2_{\bar{q}}^{-}, 3^{-}, 4_{p}^{-}, 5_{\bar{p}}^{+}, 6^{+}\right)= \\
& +\frac{[26][16]^{2}\langle 35\rangle\langle 34\rangle^{2}}{s_{345}[12]\langle 45\rangle\langle 3|4+5| 6]\langle 5|4+3| 2]} \\
& +\frac{[46][56]^{2}\langle 13\rangle\langle 23\rangle^{2}}{s_{123}\langle 12\rangle[45]\langle 3|4+5| 6]\langle 1|2+3| 4]} \\
& -\frac{\left(s_{234}\right)^{2}\langle 15\rangle[24]}{[23][34]\langle 56\rangle\langle 61\rangle\langle 1|2+3| 4]\langle 5|4+3| 2]} \text {. }
\end{aligned}
$$

The first four are computed selecting the legs as $j=6$ and $l=1$, while for the last two the chosen tags are $j=2$, $l=3$ and $j=3, l=4$, respectively.

\section{A.1.2 Sub-leading amplitudes}

The expressions for the sub-leading amplitudes are again shorter than the leading ones, reading

$$
\begin{gathered}
B_{1}=A_{6}^{(0)}\left(1_{q}^{+}, 2_{\bar{p}}^{-}, 3^{+}, 4^{-}, 5_{p}^{+}, 6_{\bar{q}}^{-}\right)= \\
-\frac{[15]^{2}\langle 24\rangle^{3}}{s_{234}\langle 23\rangle\langle 34\rangle[61]\langle 2|3+4| 5]} \\
-\frac{\langle 26\rangle^{2}[35]^{3}}{s_{345}\langle 61\rangle[34][45]\langle 2|3+4| 5]},
\end{gathered}
$$




$$
\begin{aligned}
B_{2} & =A_{6}^{(0)}\left(1_{q}^{+}, 2_{\bar{p}}^{-}, 3^{-}, 4^{+}, 5_{p}^{+}, 6_{\bar{q}}^{-}\right)= \\
& -\frac{\langle 35\rangle\langle 3|4+5| 1]^{2}}{s_{345}\langle 34\rangle\langle 45\rangle[61]\langle 5|4+3| 2]} \\
& -\frac{[24]\langle 6|2+3| 4]^{2}}{s_{234}[23][34]\langle 61\rangle\langle 5|4+3| 2]}, \\
B_{3} & =A_{6}^{(0)}\left(1_{q}^{+}, 2_{\bar{p}}^{+}, 3^{+}, 4^{-}, 5_{p}^{-}, 6_{\bar{q}}^{-}\right)= \\
& -\frac{\langle 24\rangle\langle 4|3+2| 1]^{2}}{s_{234}\langle 23\rangle\langle 34\rangle[61]\langle 2|3+4| 5]} \\
& -\frac{[35]\langle 6|5+4| 3]^{2}}{s_{345}[34][45]\langle 61\rangle\langle 2|3+4| 5]}, \\
B_{4} & =A_{6}^{(0)}\left(1_{q}^{+}, 2_{\bar{p}}^{+}, 3^{-}, 4^{+}, 5_{p}^{-}, 6_{\bar{q}}^{-}\right)= \\
& -\frac{[12]^{2}\langle 35\rangle^{3}}{s_{345}\langle 34\rangle\langle 45\rangle[61]\langle 5|4+3| 2]} \\
& -\frac{\langle 56\rangle^{2}[24]^{3}}{s_{234}[23][34]\langle 61\rangle\langle 5|4+3| 2]}, \\
& -\frac{\langle 5|6+1| 2]^{2}}{s_{234}[23][34]\langle 56\rangle\langle 61\rangle} . \\
B_{7}= & A_{6}^{(0)}\left(1_{q}^{+}, 2_{\bar{p}}^{+}, 3^{+}, 4_{p}^{-}, 5_{\bar{q}}^{-}, 6^{-}\right)= \\
B_{5}= & A_{6}^{(0)}\left(1_{q}^{+}, 2_{\bar{p}}^{-}, 3^{+}, 4_{p}^{+}, 5_{\bar{q}}^{-}, 6^{-}\right)= \\
- & \frac{\langle 2|3| 5+6| 1]^{2}}{s_{234}\langle 23\rangle\langle 34\rangle[56][61]}, \\
& =
\end{aligned}
$$

The chosen legs $(j, l)$ to apply the BCFW recursion relation were $(2,3),(3,4),(4,3),(3,4),(2,3),(6,1)$ and $(5,6)$, respectively.

\section{A.2 NMHV six-quark amplitudes}

Even though there are three color structures for the six-quark amplitudes, due to the lack of gluons the sub-subleading contributions can be directly obtained from the leading ones just by the cyclic property of the amplitudes. Therefore, there are only six independent helicity amplitudes for this process, given by the following choice

$$
\begin{array}{ll}
A_{1}=A_{6}^{(0)}\left(1_{q}^{+}, 2_{\bar{q}}^{-}, 3_{p}^{+}, 4_{\bar{p}}^{-}, 5_{r}^{+}, 6_{\bar{r}}^{-}\right) & B_{2}=A_{6}^{(0)}\left(1_{q}^{+}, 2_{\bar{p}}^{-}, 3_{p}^{+}, 4_{\bar{q}}^{-}, 5_{r}^{-}, 6_{\bar{r}}^{+}\right) \\
A_{2}=A_{6}^{(0)}\left(1_{q}^{+}, 2_{\bar{q}}^{-}, 3_{p}^{+}, 4_{\bar{p}}^{-}, 5_{r}^{-}, 6_{\bar{r}}^{+}\right) & B_{3}=A_{6}^{(0)}\left(1_{q}^{+}, 2_{\bar{p}}^{+}, 3_{p}^{-}, 4_{\bar{q}}^{-}, 5_{r}^{+}, 6_{\bar{r}}^{-}\right) \\
B_{1}=A_{6}^{(0)}\left(1_{q}^{+}, 2_{\bar{p}}^{-}, 3_{p}^{+}, 4_{\bar{q}}^{-}, 5_{r}^{+}, 6_{\bar{r}}^{-}\right) & B_{4}=A_{6}^{(0)}\left(1_{q}^{+}, 2_{\bar{p}}^{+}, 3_{p}^{-}, 4_{\bar{q}}^{-}, 5_{r}^{-}, 6_{\bar{r}}^{+}\right) .
\end{array}
$$


The two independent leading amplitudes were obtained selecting the legs as $j=2$ and $l=3$, reading

$$
\begin{aligned}
A_{1} & =A_{6}^{(0)}\left(1_{q}^{+}, 2_{\bar{q}}^{-}, 3_{p}^{+}, 4_{\bar{p}}^{-}, 5_{r}^{+}, 6_{\bar{r}}^{-}\right)= \\
& +\frac{\langle 24\rangle^{2}[15]^{2}\langle 4|3+2| 5]}{s_{234}\langle 34\rangle[56]\langle 4|3+2| 1]\langle 2|3+4| 5]} \\
& +\frac{\langle 26\rangle^{2}[35]^{2}\langle 2|5+4| 3]}{s_{345}\langle 12\rangle[34]\langle 6|1+2| 3]\langle 2|3+4| 5]} \\
& -\frac{\langle 46\rangle^{2}[13]^{2}\langle 6|3+2| 1]}{s_{123}[12]\langle 56\rangle\langle 4|3+2| 1]\langle 6|1+2| 3]},
\end{aligned}
$$

and

$$
\begin{aligned}
A_{2} & =A_{6}^{(0)}\left(1_{q}^{+}, 2_{\bar{q}}^{-}, 3_{p}^{+}, 4_{\bar{p}}^{-}, 5_{r}^{-}, 6_{\bar{r}}^{+}\right)= \\
& +\frac{\langle 24\rangle^{2}[61]^{2}\langle 4|3+2| 5]}{s_{234}\langle 34\rangle[56]\langle 4|3+2| 1]\langle 2|3+4| 5]} \\
& -\frac{\langle 2|5+4| 3]^{3}}{s_{345}\langle 12\rangle[34]\langle 2|3+4| 5]\langle 6|5+4| 3]} \\
& +\frac{\langle 45\rangle^{2}[13]^{2}\langle 6|3+2| 1]}{s_{123}[12]\langle 56\rangle\langle 4|3+2| 1]\langle 6|5+4| 3]}
\end{aligned}
$$

The sub-leading amplitudes are simply given by

$$
\begin{aligned}
B_{1} & =A_{6}^{(0)}\left(1_{q}^{+}, 2_{\bar{p}}^{-}, 3_{p}^{+}, 4_{\bar{q}}^{-}, 5_{r}^{+}, 6_{\bar{r}}^{-}\right)= \\
& +\frac{\langle 46\rangle^{2}[13]^{2}}{s_{123}[23]\langle 56\rangle\langle 4|3+2| 1]} \\
& -\frac{\langle 24\rangle^{2}[51]^{2}}{s_{234}\langle 23\rangle[56]\langle 4|3+2| 1]} \\
B_{2}=A_{6}^{(0)}\left(1_{q}^{+}, 2_{\bar{p}}^{-}, 3_{p}^{+}, 4_{\bar{q}}^{-}, 5_{r}^{-}, 6_{\bar{r}}^{+}\right)=-B_{1}(\text { flip } 5 \leftrightarrow 6) & \\
B_{3} & =A_{6}^{(0)}\left(1_{q}^{+}, 2_{\bar{p}}^{+}, 3_{p}^{-}, 4_{\bar{q}}^{-}, 5_{r}^{+}, 6_{\bar{r}}^{-}\right)= \\
& -\frac{\langle 3|2+1| 5]^{2}}{s_{123}\langle 23\rangle[56]\langle 1|2+3| 4]} \\
& +\frac{\langle 6|4+3| 2]^{2}}{s_{234}[23]\langle 56\rangle\langle 1|2+3| 4]}
\end{aligned}
$$

and

$$
B_{4}=A_{6}^{(0)}\left(1_{q}^{+}, 2_{\bar{p}}^{+}, 3_{p}^{-}, 4_{\bar{q}}^{-}, 5_{r}^{-}, 6_{\bar{r}}^{+}\right)=-B_{3}(\text { flip } 5 \leftrightarrow 6)
$$

\section{References}

[1] M. L. Mangano and S. J. Parke, Phys. Rept. 200, 301 (1991) arXiv:hep-th/0509223.

[2] L. J. Dixon, arXiv:hep-ph/9601359

[3] Z. Xu, D. H. Zhang and L. Chang, Nucl. Phys. B 291 (1987) 392.

[4] R. Kleiss and W. J. Stirling, Nucl. Phys. B 262, 235 (1985).

[5] J. F. Gunion and Z. Kunszt, Phys. Lett. B 161, 333 (1985). 
[6] F. A. Berends and W. Giele, Nucl. Phys. B 294, 700 (1987).

[7] M. L. Mangano, S. J. Parke and Z. Xu, Nucl. Phys. B 298, 653 (1988).

[8] F. Maltoni and T. Stelzer, JHEP 0302, 027 (2003) arXiv:hep-ph/0208156.

[9] E. Witten, Commun. Math. Phys. 252, 189 (2004) arXiv:hep-th/0312171.

[10] F. Cachazo, P. Svrček and E. Witten, JHEP 0409, 006 (2004) arXiv:hep-th/0403047.

[11] S. J. Parke and T. R. Taylor, Phys. Rev. Lett. 56, 2459 (1986).

[12] R. Britto, F. Cachazo and B. Feng, Nucl. Phys. B 715, 499 (2005) arXiv:hep-th/0412308.

[13] R. Britto, F. Cachazo, B. Feng and E. Witten, Phys. Rev. Lett. 94, 181602 (2005) arXiv:hep-th/0501052.

[14] G. Georgiou and V. V. Khoze, JHEP 0405 (2004) 070 arXiv:hep-th/0404072.

[15] G. Georgiou, E. W. N. Glover and V. V. Khoze, JHEP 0407 (2004) 048 arXiv:hep-th/0407027.

[16] L. J. Dixon, E. W. N. Glover and V. V. Khoze, JHEP 0412 (2004) 015 arXiv:hep-th/0411092.

[17] Z. Bern, D. Forde, D. A. Kosower and P. Mastrolia, Phys. Rev. D 72 (2005) 025006 arXiv:hep-ph/0412167.

[18] K. J. Ozeren and W. J. Stirling, JHEP 0511 (2005) 016 arXiv:hep-th/0509063.

[19] S. D. Badger, E. W. N. Glover, V. V. Khoze and P. Svrček, JHEP 0507 (2005) 025 arXiv:hep-th/0504159.

[20] J. F. Gunion and Z. Kunszt, Phys. Lett. B 159 (1985) 167 and Phys. Lett. B 176 (1986) 477.

[21] J. F. Gunion and Z. Kunszt, Phys. Lett. B 176, 163 (1986).

[22] M. x. Luo and C. k. Wen, JHEP 0503 (2005) 004 arXiv:hep-th/0501121.

[23] M. x. Luo and C. k. Wen, Phys. Rev. D 71 (2005) 091501 arXiv:hep-th/0502009.

[24] Z. Bern, V. Del Duca, L. J. Dixon and D. A. Kosower, Phys. Rev. D 71, 045006 (2005) arXiv:hep-th/0410224.

[25] R. Roiban, M. Spradlin and A. Volovich, Phys. Rev. Lett. 94, 102002 (2005) arXiv:hep-th/0412265.

[26] D. de Florian and J. Zurita, JHEP 0605 (2006) 073 arXiv:hep-ph/0605291.

[27] Z. Bern, L. J. Dixon and D. A. Kosower, Phys. Rev. D 71, 105013 (2005) arXiv:hep-th/0501240.

[28] Z. Bern, L. J. Dixon and D. A. Kosower, Phys. Rev. D 72, 125003 (2005) arXiv:hep-ph/0505055.

[29] Z. Bern, L. J. Dixon and D. A. Kosower, arXiv:hep-ph/0507005

[30] Z. Bern, N. E. J. Bjerrum-Bohr, D. C. Dunbar and H. Ita, JHEP 0511, 027 (2005) arXiv:hep-ph/0507019.

[31] Z. Bern, N. E. J. Bjerrum-Bohr, D. C. Dunbar and H. Ita, Nucl. Phys. Proc. Suppl. 157, 120 (2006) arXiv:hep-ph/0603187.

[32] C. F. Berger, Z. Bern, L. J. Dixon, D. Forde and D. A. Kosower, arXiv:hep-ph/0604195

[33] N. E. J. Bjerrum-Bohr and D. C. Dunbar, arXiv:hep-ph/0606290.

[34] C. F. Berger, Z. Bern, L. J. Dixon, D. Forde and D. A. Kosower, arXiv:hep-ph/0607014

[35] Z. G. Xiao, G. Yang and C. J. Zhu, arXiv:hep-ph/0607015

[36] X. Su, Z. G. Xiao, G. Yang and C. J. Zhu, arXiv:hep-ph/0607016.

[37] Z. G. N. Xiao, G. Yang and C. J. Zhu, arXiv:hep-ph/0607017

[38] S. D. Badger and E. W. N. Glover, arXiv:hep-ph/0607139

[39] C. Duhr, S. Hoche and F. Maltoni, arXiv:hep-ph/0607057

[40] M. Dinsdale, M. Ternick and S. Weinzierl, JHEP 0603, 056 (2006) arXiv:hep-ph/0602204.

[41] V. Del Duca, W. B. Kilgore and F. Maltoni, Nucl. Phys. B 566 (2000) 252 [Nucl. Phys. B 574 (2000) 851] arXiv:hep-ph/9910253. 